\title{
Approximation Metrics for Discrete and Continuous Systems
}

\author{
Antoine Girard and George J. Pappas, Senior Member, IEEE
}

\begin{abstract}
Established system relationships for discrete systems, such as language inclusion, simulation, and bisimulation, require system observations to be identical. When interacting with the physical world, modeled by continuous or hybrid systems, exact relationships are restrictive and not robust. In this paper, we develop the first framework of system approximation that applies to both discrete and continuous systems by developing notions of approximate language inclusion, approximate simulation, and approximate bisimulation relations. We define a hierarchy of approximation pseudo-metrics between two systems that quantify the quality of the approximation, and capture the established exact relationships as zero sections. Our approximation framework is compositional for a synchronous composition operator. Algorithms are developed for computing the proposed pseudo-metrics, both exactly and approximately. The exact algorithms require the generalization of the fixed point algorithms for computing simulation and bisimulation relations, or dually, the solution of a static game whose cost is the so-called branching distance between the systems. Approximations for the pseudo-metrics can be obtained by considering Lyapunov-like functions called simulation and bisimulation functions. We illustrate our approximation framework in reducing the complexity of safety verification problems for both deterministic and nondeterministic continuous systems.
\end{abstract}

Index Terms-Abstraction, approximation, bisimulation, metrics, transition systems.

\section{INTRODUCTION}

C OMPOSITIONAL modeling in concurrency theory [1], and complexity reduction in the formal verification of discrete systems [2] have resulted in a wealth of system relationships, including the established notions of language inclusion, simulations and bisimulations [2]. These notions have had great impact in not only reducing the complexity of discrete systems [3], but also in reducing problems for continuous and hybrid systems to purely discrete problems [4]. Much more recently, the notions of simulation and bisimulation have resulted in new equivalence notions for nondeterministic continuous [5]-[7] and hybrid systems [8]-[10].

The notions of language inclusion, simulation, and bisimulation for both discrete and continuous systems are all exact, requiring external behavior of two systems to be identical. As

Manuscript received May 17, 2005; revised April 10. 2006 and August 1, 2006. Recommended by Associate Editor A. Giua. This research was supported in part by the Région Rhône-Alpes (Projet CalCel) and the National Science Foundation Presidential Early CAREER (PECASE) under Grant 0132716.

The authors are with the Department of Electrical and Systems Engineering, the University of Pennsylvania, Philadelphia, PA 19104 USA (e-mail: agirard@ seas.upenn.edu; pappasg@seas.upenn.edu).

Color versions of one or more of the figures in this paper are available online at http://ieeexplore.ieee.org.

Digital Object Identifier 10.1109/TAC.2007.895849 exact relationships between systems might require the introduction of additional variables or states to account for errors, there are clear limitations in the amount of system compression that can be achieved. Approximate relationships which explicitly include errors, will certainly allow for more dramatic system compression. Even though this has been the tradition for deterministic continuous systems [11], it has been recently argued convincingly [12]-[14], that even for more quantitative classes of finite transition systems, such as probabilistic automata [14], labeled Markov processes [15], and quantitative transition systems [16], notions of system approximation are not only better candidates for complexity reduction but also provide more robust relationships between systems. The challenge in developing approximate system relationships is the quantification of the quality of the approximation.

The goal of this paper is to provide a theory of system approximation that applies to both finite (discrete) and infinite (continuous) transition systems by providing approximate generalizations of language inclusion, simulation, and bisimulation. By generalizing the exact notions we ensure that our framework captures the traditional exact notions for finite systems as a special case, while developing more robust notions of system approximation for infinite transition systems.

To technically achieve our goal, we consider metric transition systems, which are transition systems equipped with metrics on the state space and the observation space. Based on the observation metric, we develop a hierarchy of approximation pseudo-metrics between two metric transition systems measuring the distance from reachable set inclusion and equivalence, language inclusion and equivalence, simulation and bisimulation relations. For a large subclass of systems, the notions of exact language inclusion, simulation, and bisimulation are naturally captured as the zero sections of the pseudo-metrics. Furthermore, the relationship among the various approximation metrics is analogous to the relationship among the exact notions. For a synchronous composition operator, we show that the language, simulation and bisimulation metrics are compositional.

We then propose algorithms for computing the proposed pseudo-metrics, both exactly and approximately. Algorithms for exact computation require the generalization of the fixed point algorithms for computing simulation and bisimulation relations [17], or dually, the solution of a static game whose cost is the so-called branching distance between the systems [16]. Algorithmic relaxations for computing approximations of the pseudo-metrics can be obtained by considering Lyapunov-like functions called simulation and bisimulation functions, which are also shown to be compositional. 
This line of research has been motivated by the algorithmic verification of hybrid systems. The significant progress in the formal verification of discrete systems [3], has inspired a plethora of sophisticated methods for safety verification of continuous and hybrid systems. The approaches range from discrete and predicate abstraction methods [4], [18], [19], to reachability computations [20]-[25], to Lyapunov-like barriers [26]. However, progress on continuous (and thus hybrid) systems has been limited to systems of small continuous dimension, motivating research on model reduction [27], and projection based methods [28] for safety verification.

Since the results of this paper could be of great use in the above methods, we conclude this paper with two continuous examples that illustrate how our framework can be used in computing an over-approximation of the distance between two systems, and in reducing the complexity of safety verification for both deterministic and nondeterministic continuous systems. These examples, even though they illustrate the power of our approximation framework, are simple cases of a more systematic computational framework that is currently under development for linear systems [29], nonlinear systems [30] and hybrid systems [31].

\section{EXACT RELATIONSHIPS FOR TRANSITION SySTEMS}

\section{A. Transition Systems}

In this paper, we will consider the framework of transition systems which enables us to model in a unified way both discrete and continuous systems with either deterministic or nondeterministic dynamics (see, e.g., [5]). The results in this section can be reviewed in much greater detail in [2].

Definition 1 (Transition System): A (labeled) transition system with observations is a tuple $T=\left(Q, \Sigma, \rightarrow, Q^{0}, \Pi,\langle\langle\rangle\rangle.\right)$ that consists of

- a (possibly infinite) set $Q$ of states;

- a (possibly infinite) set $\Sigma$ of labels;

- a transition relation $\rightarrow \subseteq Q \times \Sigma \times Q$;

- a (possibly infinite) set $Q^{0} \subseteq Q$ of initial states;

- a (possibly infinite) set $\Pi$ of observations;

- an observation map $\langle\langle\rangle\rangle:. Q \rightarrow \Pi$.

The set of labeled transition systems associated to a set of labels $\Sigma$ and a set of observations $\Pi$ is denoted $\mathcal{T}(\Sigma, \Pi)$. A transition $\left(q, \sigma, q^{\prime}\right) \in \rightarrow$ will be denoted $q \stackrel{\sigma}{\rightarrow} q^{\prime}$. For simplicity, we assume that the systems we consider are nonblocking so that for all $q \in$ $Q$, there exists at least one transition $q \stackrel{\sigma}{\rightarrow} q^{\prime}$ of $T$. If for any state $q \in Q$ and any label $\sigma \in \Sigma$, there exists at most a unique transition $q \stackrel{\sigma}{\rightarrow} q^{\prime}$ of $T$ and, in addition, the set of initial states $Q^{0}$ contains a single element, then $T$ is called deterministic. Transition system $T$ is called finite if $Q$ and $\Sigma$ are finite sets, and infinite otherwise. For all labels $\sigma \in \Sigma$, the $\sigma$-successor is defined as the set valued map given by

$$
\forall q \in Q, \operatorname{Post}^{\sigma}(q)=\left\{q^{\prime} \in Q \mid q \stackrel{\sigma}{\rightarrow} q^{\prime}\right\} .
$$

We denote with $\operatorname{Supp}\left(\mathrm{Post}^{\sigma}\right)$ the support of the $\sigma$-successor which is the subset of elements $q \in Q$ such that $\operatorname{Post}^{\sigma}(q)$ is not empty. A state trajectory of $T$ is an infinite sequence of transitions

$$
q^{0} \stackrel{\sigma^{0}}{\rightarrow} q^{1} \stackrel{\sigma^{1}}{\rightarrow} q^{2} \stackrel{\sigma^{2}}{\rightarrow} \ldots \quad \text { where } q^{0} \in Q^{0} .
$$

An external trajectory is a sequence of elements of $\Pi \times \Sigma \times \Pi$ of the form

$$
\pi^{0} \stackrel{\sigma^{0}}{\rightarrow} \pi^{1} \stackrel{\sigma^{1}}{\rightarrow} \pi^{2} \stackrel{\sigma^{2}}{\rightarrow} \ldots
$$

The set of all external trajectories associated to a set of labels $\Sigma$ and a set of observations $\Pi$ is denoted $\mathcal{E}(\Sigma, \Pi)$. An external trajectory is accepted by transition system $T$ if there exists a state trajectory of $T$, such that for all $i \in \mathbb{N}, \pi^{i}=\left\langle\left\langle q^{i}\right\rangle\right\rangle$. The set of external trajectories accepted by transition system $T$ is called the language of $T$, and is denoted by $L(T)$. The reachable set of $T$ is the subset of $\Pi$ defined by

$$
\operatorname{Reach}(T)=\left\{\pi \in \Pi \mid \begin{array}{c}
\exists\left\{\pi^{i} \stackrel{\stackrel{\sigma^{i}}{\rightarrow} \pi^{i+1}}{ }\right\}_{i \in \mathbb{N}} \in L(T), \\
\exists j \in \mathbb{N}, \pi^{j}=\pi
\end{array}\right\} .
$$

One of the most important problems for transition systems is the safety verification problem which asks whether the intersection of $\operatorname{Reach}(T)$ with a set of unsafe observations $\Pi_{U} \subseteq \Pi$ is empty or not. The verification of finite transition systems of very high cardinality has motivated the development of various notion of system equivalence and system refinement that potentially reduce the complexity of safety verification [2].

\section{B. Exact Transition System Relationships}

For complexity reduction as well as for enabling compositional modeling and analysis, various notions of exact system equivalence and refinement have been established in the formal methods community [2]. In this section, we quickly review the established exact relationships in order to develop approximate versions in the subsequent sections.

Let $T_{1}=\left(Q_{1}, \Sigma_{1}, \rightarrow_{1}, Q_{1}^{0}, \Pi_{1},\langle\langle\cdot\rangle\rangle_{1}\right)$ and $T_{2}=$ $\left(Q_{2}, \Sigma_{2}, \rightarrow_{2}, Q_{2}^{0}, \Pi_{2},\langle\langle\cdot\rangle\rangle_{2}\right)$ be two labeled transition systems with the same set of labels $\left(\Sigma_{1}=\Sigma_{2}=\Sigma\right)$ and the same set of observations $\left(\Pi_{1}=\Pi_{2}=\Pi\right)$ (i.e. $T_{1}$ and $T_{2}$ are elements of $\mathcal{T}(\Sigma, \Pi)$ ).

If $L\left(T_{1}\right) \subseteq L\left(T_{2}\right)$, then it is clear from the definition of the reachable set that $\operatorname{Reach}\left(T_{1}\right) \subseteq \operatorname{Reach}\left(T_{2}\right)$. Thus, given an unsafe set $\Pi_{U}$, if $T_{2}$ is safe then $T_{1}$ is safe, since if the intersection of $\operatorname{Reach}\left(T_{2}\right)$ and $\Pi_{U}$ is empty then it follows that the intersection of $\operatorname{Reach}\left(T_{1}\right)$ and $\Pi_{U}$ is also empty. Similarly, we obtain that if $L\left(T_{1}\right)=L\left(T_{2}\right)$ then $\operatorname{Reach}\left(T_{1}\right)=\operatorname{Reach}\left(T_{2}\right)$. However, given two transition systems $T_{1}$ and $T_{2}$, checking language inclusion $\left(L\left(T_{1}\right) \subseteq L\left(T_{2}\right)\right)$ and language equivalence $\left(L\left(T_{1}\right)=L\left(T_{2}\right)\right)$ is computationally demanding for finite transition systems, and infeasible for most infinite transition systems. This has motivated the development of stronger notions of system refinement and equivalence, namely simulation and bisimulation.

Definition 2 (Simulation): A relation $\mathcal{S} \subseteq Q_{1} \times Q_{2}$ is called a simulation relation of $T_{1}$ by $T_{2}$ if for all $\left(q_{1}, q_{2}\right) \in \mathcal{S}$ :

1) $\left\langle\left\langle q_{1}\right\rangle\right\rangle_{1}=\left\langle\left\langle q_{2}\right\rangle\right\rangle_{2}$

2) $\forall q_{1} \stackrel{\sigma}{\rightarrow}_{1} q_{1}^{\prime}, \exists q_{2} \stackrel{\sigma}{\rightarrow}_{2} q_{2}^{\prime}$ such that $\left(q_{1}^{\prime}, q_{2}^{\prime}\right) \in \mathcal{S}$. 
For transition systems with a finite number of states and a finite number of labels, checking whether a relation $\mathcal{S}$ is a simulation relation is much easier (polynomial) than checking language inclusion [2].

Definition 3: $T_{2}$ simulates $T_{1}$ (denoted $T_{1} \preceq T_{2}$ ) if there exists $\mathcal{S}$, a simulation relation of $T_{1}$ by $T_{2}$, such that for all $q_{1} \in Q_{1}^{0}$, there exists $q_{2} \in Q_{2}^{0}$ such that $\left(q_{1}, q_{2}\right) \in \mathcal{S}$.

Note that the relation $\preceq$ is a preorder on the set $\mathcal{T}(\Sigma, \Pi)$ of transition systems. An interesting case is when a relation is a simulation of $T_{1}$ by $T_{2}$ as well as a simulation of $T_{2}$ by $T_{1}$. Such a relation is called a bisimulation.

Definition 4 (Bisimulation): A relation $\mathcal{B} \subseteq Q_{1} \times Q_{2}$ is called a bisimulation relation between $T_{1}$ and $T_{2}$ if for all $\left(q_{1}, q_{2}\right) \in \mathcal{B}$

1) $\left\langle\left\langle q_{1}\right\rangle\right\rangle_{1}=\left\langle\left\langle q_{2}\right\rangle\right\rangle_{2}$;

2) $\forall q_{1} \underset{\sigma_{\sigma}}{\rightarrow} q_{1}^{\prime}, \exists q_{2} \underset{\sigma_{\sigma}}{\rightarrow} q_{2}^{\prime}$ such that $\left(q_{1}^{\prime}, q_{2}^{\prime}\right) \in \mathcal{B}$;

3) $\forall q_{2} \stackrel{\sigma}{\rightarrow}_{2} q_{2}^{\prime}, \exists q_{1} \stackrel{\sigma}{\rightarrow}_{1} q_{1}^{\prime}$ such that $\left(q_{1}^{\prime}, q_{2}^{\prime}\right) \in \mathcal{B}$.

If any initial state of $T_{1}$ can be related to an initial state of $T_{2}$ and conversely, then $T_{1}$ and $T_{2}$ simulate each other. We say that $T_{1}$ and $T_{2}$ are bisimilar.

Definition 5: $T_{1}$ and $T_{2}$ are bisimilar (denoted $T_{1} \cong T_{2}$ ) if there exists $\mathcal{B}$, a bisimulation relation between $T_{1}$ and $T_{2}$ such that, for all $q_{1} \in Q_{1}^{0}$, there exists $q_{2} \in Q_{2}^{0}$ such that $\left(q_{1}, q_{2}\right) \in \mathcal{B}$ and conversely.

The relation $\cong$ is an equivalence relation on the set of transition systems $\mathcal{T}(\Sigma, \Pi)$. Bisimulations have been vital in collapsing infinite transition systems to bisimilar finite transition systems, especially in the context of timed and hybrid systems [4]. The different relationships between transition systems are summarized in the following classical result:

Theorem 1 (Hierarchy of Relationships): For all transition systems $T_{1}, T_{2} \in \mathcal{T}(\Sigma, \Pi)$,

$\begin{array}{ccc}T_{1} \cong T_{2} & \Rightarrow L\left(T_{1}\right)=L\left(T_{2}\right) \Rightarrow & \operatorname{Reach}\left(T_{1}\right)=\operatorname{Reach}\left(T_{2}\right) \\ \Downarrow & \Downarrow & \\ T_{1} \preceq T_{2} & \Rightarrow L\left(T_{1}\right) \subseteq L\left(T_{2}\right) \Rightarrow & \operatorname{Reach}\left(T_{1}\right) \subseteq \operatorname{Reach}\left(T_{2}\right) .\end{array}$

Let us remark that if $T_{1}$ and $T_{2}$ are bisimilar then solving the reachability problem for $T_{1}$ is equivalent to solving the reachability problem for $T_{2}$. Even though from a verification perspective we would like to relate the reachable sets of transition systems, complexity considerations force us to consider stronger relationships between transition systems. However, it is well known that the notions of simulation and bisimulation are different than language inclusion or language equality only for nondeterministic transition systems [1]. For deterministic labeled transition systems, the notions become equivalent.

Theorem 2: If $T_{1}$ and $T_{2}$ are deterministic, then the following equivalences hold

$$
\begin{aligned}
& T_{1} \cong T_{2} \quad \Longleftrightarrow \quad L\left(T_{1}\right)=L\left(T_{2}\right) \\
& T_{1} \preceq T_{2} \quad \Longleftrightarrow \quad L\left(T_{1}\right) \subseteq L\left(T_{2}\right) .
\end{aligned}
$$

The fact that, in the presence of nondeterminism, simulation and bisimulation are stronger than language (or trajectory) equivalence has resulted in novel notions of exact system equivalence for nondeterministic dynamical, control, and hybrid systems [5]-[10].

\section{Metric Transition Systems}

As exact relationships between transition systems do not permit any error, there are clear limitations in the amount of system compression that can be achieved. Approximate relationships which do allow for the possibility of error, will certainly allow for more dramatic system compression. Even though this has been the tradition for deterministic continuous systems [11], it has been recently argued convincingly that even for more quantitative classes of finite transition systems, such as probabilistic automata [14], labeled Markov processes [15], and quantitative transition systems [16], notions of system approximation are not only better candidates for complexity reduction but also provide more robust relationships between systems. The challenge of approximate system relationships is the quantification of the quality of the approximation.

The goal of this paper is to provide a theory of system approximation that applies to both finite (discrete) and infinite (continuous) transition systems, by providing approximate generalizations of the exact relationships of Section II-B. By generalizing the exact notions we ensure that our framework captures the traditional exact notions for finite systems as a special case, while developing more robust notions of system approximation for infinite transition systems. To technically achieve our goal, we must equip the transition systems we consider with some topological structure that is induced by metrics on the state space and the observation space.

Definition 6(Metric Transition Systems): A transition system $T=\left(Q, \Sigma, \rightarrow, Q^{0}, \Pi,\langle\langle\cdot\rangle\rangle\right)$ is called a metric transition system if $\left(Q, d_{Q}\right)$ and $\left(\Pi, d_{\Pi}\right)$ are metric spaces. The set of metric transition systems associated to a set of labels $\Sigma$ and a set of observations $\Pi$ is denoted $\mathcal{T}_{M}(\Sigma, \Pi)$.

Note that, in this paper, we do not equip the set of labels $\Sigma$ with any metric (equivalently we consider $\Sigma$ with the trivial discrete metric). In this paper, we also need to distinguish a special class of metric transition systems that enjoy some additional regularity assumptions.

Definition 7 (Regular Metric Transition Systems): A metric transition system $T \in \mathcal{T}_{M}(\Sigma, \Pi)$ is called regular if

1) its set of initial values $Q_{0}$ is compact;

2) its observation map $\langle\langle\rangle$.$\rangle is continuous;$

3 ) its transition relation satisfies the following properties:

a) for all $\sigma \in \Sigma$, the set valued map Post $^{\sigma}$ is continuous; 1

b) for all $\sigma \in \Sigma$, $\operatorname{Supp}\left(\operatorname{Post}^{\sigma}\right)$ is an open subset of $Q$;

c) for all $\sigma \in \Sigma$, for all $q \in \operatorname{Supp}\left(\operatorname{Post}^{\sigma}\right), \operatorname{Post}^{\sigma}(q)$ is a compact subset of $Q$

d) for all $\sigma \in \Sigma$, for all $q \in \operatorname{Supp}\left(\operatorname{Post}^{\sigma}\right)$, $\operatorname{Post}^{\sigma}(q)$ has a compact neighborhood.

The set of regular metric transition systems is denoted $\mathcal{T}_{M}^{*}(\Sigma, \Pi)$.

Remark 1: For usual metric spaces such as finite dimensional vector spaces, Property (3.d) is a direct consequence of the property (3.c). However, as noted in [32], it is not necessarily the case when we consider some infinite dimensional metric spaces such as the functional space $L^{2}$. Such metric spaces arise if the transition system is derived from partial differential equations.

\footnotetext{
${ }^{1}$ Set-valued continuity concepts are stated in Appendix.
} 
Let us present some broad classes of regular metric transition systems that are of great interest in this paper. In particular, we are interested in finite transition systems as models of discrete systems, and infinite transition systems as models of continuous systems.

\section{A. Finite Transition Systems}

If $Q$ is a finite set, then for any metrics defined on $Q$ and $\Pi$, it is easy to check that the properties of Definition 7 hold. This example, although trivial, ensures that the framework developed in this paper will apply and capture the existing exact relationships for purely discrete systems.

\section{B. Continuous Dynamical Systems}

Let us consider the following differential inclusion:

$$
\begin{cases}\dot{x}(t) \in F(x(t)), & x(0) \in I, x(t) \in \mathbb{R}^{n} \\ y(t)=g(x(t)), & y(t) \in \mathbb{R}^{p}\end{cases}
$$

where $F$ is a set valued map. This framework includes ordinary differential equations as well as control systems [33]. Following [5], we can derive a nondeterministic labeled transition system $T=\left(Q, \Sigma, \rightarrow, Q^{0}, \Pi,\langle\langle\cdot\rangle\rangle\right)$ from this differential inclusion by the following procedure:

- the set of states is $Q=\mathbb{R}^{n}$;

- the labels stand for the time, i.e. $\Sigma=\mathbb{R}^{+}$;

- the transition relation is given by $q \stackrel{t}{\rightarrow} q^{\prime}$ if and only if there exists a function $x($.$) such that x(0)=q, x(t)=q^{\prime}$ and for almost all $s \in[0, t], \dot{x}(s) \in F(x(s))$;

- the set of initial values is $Q^{0}=I$;

- the set of observations is $\Pi=\mathbb{R}^{p}$;

- the observation map is given by $\langle\langle x\rangle\rangle=g(x)$.

Let us assume that $I$ is compact and $g$ is continuous. If in addition the set valued map $F$ is continuous, has compact convex images and Lipschitz, that is

$$
\exists L, \forall x_{1} \in \mathbb{R}^{n}, \forall x_{2} \in \mathbb{R}^{n} \quad h\left(F\left(x_{1}\right), F\left(x_{2}\right)\right) \leq L\left\|x_{1}-x_{2}\right\|
$$

where $h$ denotes the Hausdorff distance (see Appendix for a quick review) then we can show [33] that the defined transition system satisfies the conditions of Definition 7.

\section{ApProximation Metrics for Metric TRANSITION SYSTEMS}

Metric transition systems have enough structure to develop a hierarchy of system approximation metrics, eventually resulting in an approximate version of Theorem 1 . We begin with notions of approximate reachability and approximate language inclusion, and continue with the stronger notions of approximate simulation and bisimulation.

\section{A. Reachability and Language Metrics}

Since the set of observations is now a metric space $\left(\Pi, d_{\Pi}\right)$, we can denote by $h_{\Pi}$ and $h_{\Pi}$ respectively the directed and undirected Hausdorff distances associated to the metric $d_{\Pi}$. The reachability metric between $T_{1}$ and $T_{2}$ is naturally defined as the Hausdorff distance between $\operatorname{Reach}\left(T_{1}\right)$ and $\operatorname{Reach}\left(T_{2}\right)$.
Definition 8 (Reachability Metrics): The directed and undirected reachability metrics are defined respectively as

$$
\begin{aligned}
& d_{\mathcal{R}}\left(T_{1}, T_{2}\right)=h_{\Pi}\left(\operatorname{Reach}\left(T_{1}\right), \operatorname{Reach}\left(T_{2}\right)\right) \\
& d_{\mathcal{R}}\left(T_{1}, T_{2}\right)=h_{\Pi}\left(\operatorname{Reach}\left(T_{1}\right), \operatorname{Reach}\left(T_{2}\right)\right) .
\end{aligned}
$$

Since the reachability metrics are Hausdorff distances, the following result is a direct consequence of the well-known properties of Hausdorff distances.

Theorem 3: The reachability metrics are pseudo-metrics on the set of metric transition systems $\mathcal{T}_{M}(\Sigma, \Pi)$ and

$$
\begin{aligned}
d_{\mathcal{R}}\left(T_{1}, T_{2}\right) & =0 \quad \Longleftrightarrow \operatorname{cl}\left(\operatorname{Reach}\left(T_{1}\right)\right) \subseteq \operatorname{cl}\left(\operatorname{Reach}\left(T_{2}\right)\right) \\
d_{\mathcal{R}}\left(T_{1}, T_{2}\right)=0 & \Longleftrightarrow \operatorname{cl}\left(\operatorname{Reach}\left(T_{1}\right)\right)=\operatorname{cl}\left(\operatorname{Reach}\left(T_{2}\right)\right)
\end{aligned}
$$

where $\operatorname{cl}($.$) denotes the closure of a set.$

For safety verification, the reachability metric is of great interest. Indeed, if we could compute $d_{\overrightarrow{\mathcal{R}}}\left(T_{1}, T_{2}\right)$ we would have that

$$
\operatorname{Reach}\left(T_{1}\right) \subseteq N\left(\operatorname{cl}\left(\operatorname{Reach}\left(T_{2}\right)\right), d_{\overrightarrow{\mathcal{R}}}\left(T_{1}, T_{2}\right)\right)
$$

where $N(\pi, \delta)$ denotes the $\delta$ neighborhood of $\pi \in \Pi$. Hence, if the distance separating $\operatorname{Reach}\left(T_{2}\right)$ and the unsafe set $\Pi_{U}$ is strictly greater than $d_{\overrightarrow{\mathcal{R}}}\left(T_{1}, T_{2}\right)$, then the intersection of $\operatorname{Reach}\left(T_{1}\right)$ and $\Pi_{U}$ is empty and therefore $T_{1}$ is safe.

Unfortunately the reachability metric is impossible to compute exactly for most infinite metric transition systems, and extremely difficult for most finite transition systems. We will therefore develop a hierarchy of stronger metrics, starting with two metrics that measure the distance between the languages of two systems. In order to define a distance between two languages, we first have to consider a metric in the space of external trajectories. Let $s_{1}$ and $s_{2}$ be two elements of $\mathcal{E}(\Sigma, \Pi)$

$$
s_{1}=\left\{\pi_{1}^{i} \stackrel{\sigma_{1}^{i}}{\rightarrow} \pi_{1}^{i+1}\right\}_{i \in \mathbb{N}} \quad s_{2}=\left\{\pi_{2}^{i} \stackrel{\sigma_{2}^{i}}{\rightarrow} \pi_{2}^{i+1}\right\}_{i \in \mathbb{N}} .
$$

Since we are interested in safety verification problems, it makes sense to define the distance between $s_{1}$ and $s_{2}$ as

$$
d_{\mathcal{E}}\left(s_{1}, s_{2}\right)= \begin{cases}\sup _{i \in \mathbb{N}} d_{\Pi}\left(\pi_{1}^{i}, \pi_{2}^{i}\right), & \text { if } \forall j \in \mathbb{N}, \sigma_{1}^{j}=\sigma_{2}^{j} \\ +\infty, & \text { otherwise. }\end{cases}
$$

Proposition 1: $d_{\mathcal{E}}$ is a metric on the set of external trajectories $\mathcal{E}(\Sigma, \Pi)$.

The proof is quite straightforward and can be found in [34].

Let $h_{\mathcal{E}}$ and $h_{\mathcal{E}}$ denote respectively the directed and undirected Hausdorff distance associated to the metric $d_{\mathcal{E}}$. Since $L\left(T_{1}\right)$ and $L\left(T_{2}\right)$ are subsets of $\mathcal{E}(\Sigma, \Pi)$, the language metric between $T_{1}$ and $T_{2}$ can then be defined as the Hausdorff distance between the languages $L\left(T_{1}\right)$ and $L\left(T_{2}\right)$.

Definition 9 (Language Metrics): The directed and undirected language metrics are defined, respectively, as

$$
\begin{aligned}
d_{\overrightarrow{\mathcal{L}}}\left(T_{1}, T_{2}\right) & =h_{\overrightarrow{\mathcal{E}}}\left(L\left(T_{1}\right), L\left(T_{2}\right)\right) \\
d_{\mathcal{L}}\left(T_{1}, T_{2}\right) & =h_{\mathcal{E}}\left(L\left(T_{1}\right), L\left(T_{2}\right)\right) .
\end{aligned}
$$

The intuitive meaning of the directed language metric is the following. For any external trajectory of the system $T_{1}$, we can find an external trajectory of the system $T_{2}$, with the same sequence 
of labels, such that the distance between the observations of the two systems remains bounded by $d_{\mathcal{L}}\left(T_{1}, T_{2}\right)$.

Similar to the reachability metrics, the following result follows as a consequence of the properties of Hausdorff distances.

Theorem 4: The language metrics are pseudo-metrics on the set of metric labeled transition systems $\mathcal{T}_{M}(\Sigma, \Pi)$ and

$$
\begin{aligned}
& d_{\mathcal{L}}\left(T_{1}, T_{2}\right)=0 \Longleftrightarrow \operatorname{cl}\left(L\left(T_{1}\right)\right) \subseteq \operatorname{cl}\left(L\left(T_{2}\right)\right) \\
& d_{\mathcal{L}}\left(T_{1}, T_{2}\right)=0 \Longleftrightarrow \operatorname{cl}\left(L\left(T_{1}\right)\right)=\operatorname{cl}\left(L\left(T_{2}\right)\right) \text {. }
\end{aligned}
$$

The following inequalities hold between the reachability and language metrics.

Lemma 1: For all $T_{1}, T_{2} \in \mathcal{T}_{M}(\Sigma, \Pi), d_{\mathcal{R}}\left(T_{1}, T_{2}\right) \leq$ $d_{\mathcal{L}}\left(T_{1}, T_{2}\right)$ and $d_{\mathcal{R}}\left(T_{1}, T_{2}\right) \leq d_{\mathcal{L}}\left(T_{1}, T_{2}\right)$.

Proof: Let $\varepsilon>0$. Let $\pi_{1}$ be an element of $\operatorname{Reach}\left(T_{1}\right)$. There exists an external trajectory of $T_{1}$

$$
s_{1}=\left\{\pi_{1}^{i} \stackrel{\sigma_{1}^{i}}{\rightarrow} \pi_{1}^{i+1}\right\}_{i \in \mathbb{N}}
$$

such that $\pi_{1}^{j}=\pi_{1}$ for some $j \in \mathbb{N}$. There also exists an external trajectory of $T_{2}$,

$$
s_{2}=\left\{\pi_{2}^{i} \stackrel{\sigma_{2}^{i}}{\rightarrow} \pi_{2}^{i+1}\right\}_{i \in \mathbb{N}}
$$

such that $d_{\mathcal{E}}\left(s_{1}, s_{2}\right)<d_{\mathcal{L}}\left(T_{1}, T_{2}\right)+\varepsilon$. Particularly, this means that $d_{\Pi}\left(\pi_{1}^{j}, \pi_{2}^{j}\right)<d_{\mathcal{L}}\left(T_{1}, T_{2}\right)+\varepsilon$. Since $\pi_{2}^{j}$ is an element of $\operatorname{Reach}\left(T_{2}\right)$, we have $d_{\mathcal{R}}\left(T_{1}, T_{2}\right)<d_{\mathcal{L}}\left(T_{1}, T_{2}\right)+\varepsilon$. This holds for all $\varepsilon>0$, hence $d_{\mathcal{R}}\left(T_{1}, T_{2}\right) \leq d_{\mathcal{L}}\left(T_{1}, T_{2}\right)$. The inequality for the undirected metric is straightforward.

The computation of $d_{\mathcal{L}}\left(T_{1}, T_{2}\right)$ and $d_{\mathcal{L}}\left(T_{1}, T_{2}\right)$ is also extremely difficult (but feasible in the case of quantitative, finite transition systems [16]). We will therefore consider approximate versions of the stronger notions of simulation and bisimulation.

\section{B. Approximate Simulation and Simulation Metric}

1) Approximate Simulation: We introduce a notion of approximate simulation that is obtained by relaxing the exact observational equivalence required by exact simulation relations. Instead of requiring that the observations of two systems start and remain identical, we require that they start and remain close.

Definition 10 (Approximate Simulation): Let $T_{1}, T_{2} \in$ $\mathcal{T}_{M}(\Sigma, \Pi)$. A relation $\mathcal{S}_{\delta} \subseteq Q_{1} \times Q_{2}$ is called a $\delta$-approximate simulation relation of $T_{1}$ by $T_{2}$ if for all $\left(q_{1}, q_{2}\right) \in \mathcal{S}_{\delta}$

1) $d_{\Pi}\left(\left\langle\left\langle q_{1}\right\rangle\right\rangle_{1},\left\langle\left\langle q_{2}\right\rangle\right\rangle_{2}\right) \leq \delta$

2) $\forall q_{1} \stackrel{\sigma}{\rightarrow}_{1} q_{1}^{\prime}, \exists q_{2} \stackrel{\sigma}{\rightarrow}_{2} q_{2}^{\prime}$ such that $\left(q_{1}^{\prime}, q_{2}^{\prime}\right) \in \mathcal{S}_{\delta}$.

Since $d_{\Pi}$ is a metric, for $\delta=0$ we recover the established definition of exact simulation relation. Parameter $\delta$ can serve as a measure of simulation precision.

Definition 11: Transition system $T_{2}$ approximately simulates $T_{1}$ with precision $\delta$ (noted $T_{1} \preceq_{\delta} T_{2}$ ), if there exists $\mathcal{S}_{\delta}$, a $\delta$-approximate simulation relation of $T_{1}$ by $T_{2}$ such that for all $q_{1} \in Q_{1}^{0}$, there exists $q_{2} \in Q_{2}^{0}$ such that $\left(q_{1}, q_{2}\right) \in \mathcal{S}_{\delta}$.

The following result ensures that the set of $\delta$-approximate simulation relations has a maximal element.
Lemma 2: Let $\left\{\mathcal{S}_{\delta}^{i}\right\}_{i \in I}$ be a (possibly uncountable) family of $\delta$-approximate simulation relations of $T_{1}$ by $T_{2}$. Then, $\bigcup_{i \in I} \mathcal{S}_{\delta}^{i}$ is a $\delta$-approximate simulation relation of $T_{1}$ by $T_{2}$.

Proof: Let $\left(q_{1}, q_{2}\right) \in \bigcup_{i \in I} \mathcal{S}_{\delta}^{i}$, there exists $i \in I$ such that $\left(q_{1}, q_{2}\right) \in \mathcal{S}_{\delta}^{i}$. Then, $d_{\Pi}\left(\left\langle\left\langle q_{1}\right\rangle\right\rangle_{1},\left\langle\left\langle q_{2}\right\rangle\right\rangle_{2}\right) \leq \delta$. Moreover, for all $q_{1} \stackrel{\sigma}{\rightarrow} 1 q_{1}^{\prime}$, there exists $q_{2} \stackrel{\sigma}{\rightarrow}_{2} q_{2}^{\prime}$ such that $\left(q_{1}^{\prime}, q_{2}^{\prime}\right) \in \mathcal{S}_{\delta}^{i} \subseteq$ $\bigcup_{i \in I} \mathcal{S}_{\delta}^{i}$.

Given a precision parameter $\delta$, Lemma 2 allows us to define the largest simulation relation between two systems.

Definition 12: Let $\left\{\mathcal{S}_{\delta}^{i}\right\}_{i \in I}$ be the set of $\delta$-approximate simulation relations of $T_{1}$ by $T_{2}$. The maximal $\delta$-approximate simulation relation of $T_{1}$ by $T_{2}$ is defined by

$$
\mathcal{S}_{\delta}^{\max }=\bigcup_{i \in I} \mathcal{S}_{\delta}^{i}
$$

It is clear that $T_{2}$ approximately simulates $T_{1}$ with precision $\delta$ if and only if for all $q_{1} \in Q_{1}^{0}$, there exists $q_{2} \in Q_{2}^{0}$ such that $\left(q_{1}, q_{2}\right) \in \mathcal{S}_{\delta}^{\max }$. Approximate simulation relations define a parameterized family of relations on the set of metric transition systems $\mathcal{T}_{M}(\Sigma, \Pi)$. These relations satisfy the following properties:

Proposition 2: Let $T_{1}, T_{2}$ and $T_{3} \in \mathcal{T}_{M}(\Sigma, \Pi)$

1) For all $\delta \geq 0, T_{1} \preceq_{\delta} T_{1}$;

2) For all $\delta \geq 0$, if $T_{1} \preceq \delta T_{2}$, then for all $\delta^{\prime} \geq \delta, T_{1} \preceq \delta_{\delta^{\prime}} T_{2}$;

3) For all $\delta \geq 0, \delta^{\prime} \geq 0$, if $T_{1} \preceq \delta T_{2}$ and $T_{2} \preceq \delta^{\prime} T_{3}$, then $T_{1} \preceq \delta+\delta^{\prime} T_{3}$.

Proof: The first property is obvious. Let us remark that a $\delta$-approximate simulation relation of $T_{1}$ by $T_{2}$ is also a $\delta^{\prime}$-approximate simulation relation of $T_{1}$ by $T_{2}$ (for $\delta^{\prime} \geq \delta$ ); the second property is straightforward. $T_{1} \preceq_{\delta} T_{2}$, let $S_{\delta}^{\max }$ be the maximal $\delta$-approximate simulation relation of $T_{1}$ by $T_{2}$. $T_{2} \preceq_{\delta^{\prime}} T_{3}$, let $S_{\delta^{\prime}}^{\max }$ be the maximal $\delta^{\prime}$-approximate simulation relation of $T_{2}$ by $T_{3}$. Let us define the following relation $\mathcal{S}_{\delta+\delta^{\prime}} \subseteq Q_{1} \times Q_{3}$ :

$$
\mathcal{S}_{\delta+\delta^{\prime}}=\left\{\left(q_{1}, q_{3}\right) \mid \begin{array}{l}
\exists q_{2} \in Q_{2} \text { such that } \\
\left(q_{1}, q_{2}\right) \in \mathcal{S}_{\delta}^{\max } \text { and }\left(q_{2}, q_{3}\right) \in \mathcal{S}_{\delta^{\prime}}^{\max }
\end{array}\right\} .
$$

Let $\left(q_{1}, q_{3}\right) \in \mathcal{S}_{\delta+\delta^{\prime}}$, let $q_{2}$ be the corresponding element of $Q_{2}$

$$
\begin{aligned}
d_{\Pi}\left(\left\langle\left\langle q_{1}\right\rangle\right\rangle_{1},\left\langle\left\langle q_{3}\right\rangle\right\rangle_{3}\right) \leq & d_{\Pi}\left(\left\langle\left\langle q_{1}\right\rangle\right\rangle_{1},\left\langle\left\langle q_{2}\right\rangle\right\rangle_{2}\right) \\
& +d_{\Pi}\left(\left\langle\left\langle q_{2}\right\rangle\right\rangle_{2},\left\langle\left\langle q_{3}\right\rangle\right\rangle_{3}\right) \\
\leq & \delta+\delta^{\prime}
\end{aligned}
$$

For all $q_{1} \stackrel{\sigma}{\rightarrow} 1 q_{1}^{\prime}$, there exists $q_{2} \stackrel{\sigma}{\rightarrow}{ }_{2} q_{2}^{\prime}$ such that $\left(q_{1}^{\prime}, q_{2}^{\prime}\right) \in$ $\mathcal{S}_{\delta}^{\max }$, there also exists $q_{3} \stackrel{\sigma}{\rightarrow} 3 q_{3}^{\prime}$ such that $\left(q_{2}^{\prime}, q_{3}^{\prime}\right) \in \mathcal{S}_{\delta^{\prime}}^{\max }$. Hence, $\left(q_{1}^{\prime}, q_{3}^{\prime}\right) \in \mathcal{S}_{\delta+\delta^{\prime}}$. Therefore, $\mathcal{S}_{\delta+\delta^{\prime}}$ is a $\left(\delta+\delta^{\prime}\right)$-approximate simulation relation of $T_{1}$ by $T_{3}$. Moreover, for all $q_{1} \in Q_{1}^{0}$, there exists $q_{2} \in Q_{2}^{0}$ such that $\left(q_{1}, q_{2}\right) \in \mathcal{S}_{\delta}^{\max }$, there also exists $q_{3} \in Q_{3}^{0}$ such that $\left(q_{2}, q_{3}\right) \in \mathcal{S}_{\delta^{\prime}}^{\max }$. Therefore, $T_{1} \preceq \delta+\delta^{\prime} T_{2}$.

Let us remark that contrary to the relation $\preceq$, the relation $\preceq_{\delta}$ (for $\delta>0$ ) is not a preorder ${ }^{2}$ on the set of metric transition systems $\mathcal{T}_{M}(\Sigma, \Pi)$. Indeed, the third property of Proposition 2 is not a transitivity property. However, it can be interpreted as a

${ }^{2}$ However, the relation $T_{1} \lesssim T_{2}$ defined as $\exists \delta: T_{1} \preceq_{\delta} T_{2}$ is a preorder in $\mathcal{T}_{M}(\Sigma, \Pi)$. 
triangular inequality and, therefore, the precision of the approximate simulation of $T_{1}$ by $T_{2}$ appears to be a good criterion to define a distance between the two systems.

2) Simulation Metric: The simulation metric is defined as the tightest precision $\delta$ with which $T_{2}$ approximately simulates $T_{1}$.

Definition 13 (Simulation Metric): The simulation metric is defined by

$$
d_{\mathcal{S}}\left(T_{1}, T_{2}\right)=\inf \left\{\delta \mid T_{1} \preceq{ }_{\delta} T_{2}\right\} .
$$

Theorem 5: The simulation metric is a directed pseudo metric on the set of metric labeled transition systems $\mathcal{T}_{M}(\Sigma, \Pi)$ and

$$
T_{1} \preceq T_{2} \Longrightarrow d_{\mathcal{S}}\left(T_{1}, T_{2}\right)=0 .
$$

Proof: Let $T_{1}, T_{2}$ and $T_{3}$ be elements of $\mathcal{T}_{M}(\Sigma, \Pi)$. Let us remark that from Proposition 2, we have the following inclusion:

$$
\left\{\delta+\delta^{\prime} \mid T_{1} \preceq_{\delta} T_{2} \text { and } T_{2} \preceq_{\delta^{\prime}} T_{3}\right\} \subseteq\left\{\delta \mid T_{1} \preceq_{\delta} T_{3}\right\} .
$$

Hence

$$
\begin{aligned}
d_{\mathcal{S}}\left(T_{1}, T_{3}\right) & =\inf \left\{\delta \mid T_{1} \preceq_{\delta} T_{3}\right\} \\
& \leq \inf \left\{\delta+\delta^{\prime} \mid T_{1} \preceq_{\delta} T_{2} \text { and } T_{2} \preceq_{\delta^{\prime}} T_{3}\right\} \\
& \leq \inf \left\{\delta \mid T_{1} \preceq_{\delta} T_{2}\right\}+\inf \left\{\delta \mid T_{2} \preceq_{\delta} T_{3}\right\} \\
& \leq d_{\mathcal{S}}\left(T_{1}, T_{2}\right)+d_{\mathcal{S}}\left(T_{2}, T_{3}\right) .
\end{aligned}
$$

Therefore, the triangular inequality holds. The second part of the proposition is obvious.

A counter-example showing that the converse direction of Theorem 5 does not hold for the general class of metric transition systems $\mathcal{T}_{M}(\Sigma, \Pi)$ can be found in [34]. The converse direction of Theorem requires the development of some topological results about simulation relations that needs the additional structure of regular metric transitions systems $\mathcal{T}_{M}^{*}(\Sigma, \Pi)$.

Lemma 3: Let $T_{1}, T_{2} \in \mathcal{T}_{M}^{*}(\Sigma, \Pi)$, let $\mathcal{R} \subseteq Q_{1} \times Q_{2}$ be a closed subset then

$$
\mathcal{R}^{\prime}=\left\{\left(q_{1}, q_{2}\right) \in \mathcal{R} \mid \forall q_{1} \stackrel{\sigma}{\rightarrow}_{1} q_{1}^{\prime}, \exists q_{2} \stackrel{\sigma}{\rightarrow}_{2} q_{2}^{\prime},\left(q_{1}^{\prime}, q_{2}^{\prime}\right) \in \mathcal{R}\right\}
$$

is a closed subset as well.

Proof: Let $\left(q_{1}, q_{2}\right) \in \operatorname{cl}\left(\mathcal{R}^{\prime}\right)$, there exists a sequence $\left\{\left(q_{1}^{i}, q_{2}^{i}\right)\right\}_{i \in \mathbb{N}}$ of elements of $\mathcal{R}^{\prime}$ converging to $\left(q_{1}, q_{2}\right)$. First, let us remark that since $\mathcal{R}$ is closed, $\left(q_{1}, q_{2}\right) \in \mathcal{R}$. Let $q_{1} \stackrel{\sigma}{\rightarrow} q_{1}^{\prime}$ (i.e. $q_{1}^{\prime} \in \operatorname{Post}_{1}^{\sigma}\left(q_{1}\right)$ ), since the support of the $\sigma$-successor is open, there exists $n \in \mathbb{N}$, such that for all $i \geq n$, $q_{1}^{i} \in \operatorname{Supp}\left(\operatorname{Post}_{1}^{\sigma}\right)$. The set valued map Post ${ }_{1}^{\sigma}$ is lower semicontinuous, hence there exists a sequence $\left\{q_{1}^{i i}\right\}_{i \geq n}$ such that for all $i \geq n, q_{1}^{\prime i} \in \operatorname{Post}_{1}^{\sigma}\left(q_{1}^{i}\right)$ and which converges to $q_{1}^{\prime}$. Since $\left(q_{1}^{i}, q_{2}^{i}\right)$ is in $\mathcal{R}^{\prime}$, then for all $i \geq n$, there exists $q_{2}^{\prime i} \in \operatorname{Post}_{2}^{\sigma}\left(q_{2}^{i}\right)$ such that $\left(q_{1}^{\prime i}, q_{2}^{\prime i}\right) \in \mathcal{R}$. By assumption, the set $\operatorname{Post}_{2}^{\sigma}\left(q_{2}\right)$ has a compact neighborhood $V$. Since Post $_{2}^{\sigma}$ is upper semicontinuous and since $\left\{q_{2}^{i}\right\}_{i \in N}$ converges to $q_{2}$, there exists $m \geq n$ such that for all $i \geq m, q_{2}^{\prime i} \in \operatorname{Post}_{2}^{\sigma}\left(q_{2}^{i}\right) \subseteq V$. $V$ is a compact, hence there exists a subsequence of the sequence $\left\{q_{2}^{\prime i}\right\}_{i \geq m}$ which we will also note $\left\{q_{2}^{\prime i}\right\}_{i \geq m}$ and which converges to a limit $q_{2}^{\prime} \in V$. Now, for all neighborhood $W$ of $\operatorname{Post}_{2}^{\sigma}\left(q_{2}\right)(W \subseteq V)$, there exists $p \geq m$ such that for all $i \geq p, q_{2}^{\prime i} \in \operatorname{Post}_{2}^{\sigma}\left(q_{2}^{i}\right) \subseteq W$. Hence $q_{2}^{\prime} \in \operatorname{cl}(W)$. Since this holds for all neighborhood of Post ${ }_{2}^{\sigma}\left(q_{2}\right)$ we have $q_{2}^{\prime} \in \operatorname{cl}\left(\operatorname{Post}_{2}^{\sigma}\left(q_{2}\right)\right)=\operatorname{Post}_{2}^{\sigma}\left(q_{2}\right)$ because $\operatorname{Post}_{2}^{\sigma}\left(q_{2}\right)$ is compact. Hence, we have $q_{2} \stackrel{\sigma}{\rightarrow}{ }_{2} q_{2}^{\prime}$. $\left(q_{1}^{\prime}, q_{2}^{\prime}\right)$ is the limit of a sequence of elements of the closed subset $\mathcal{R}$, therefore $\left(q_{1}^{\prime}, q_{2}^{\prime}\right) \in \mathcal{R}$. Hence, $\left(q_{1}, q_{2}\right) \in \mathcal{R}^{\prime}$ which is consequently closed.

A consequence of Lemma 3 is the following.

Proposition 3: Let $T_{1}, T_{2} \in \mathcal{T}_{M}^{*}(\Sigma, \Pi)$, and let $\mathcal{S}_{\delta}$ be a $\delta$-approximate simulation relation of $T_{1}$ by $T_{2}$. Then $\operatorname{cl}\left(\mathcal{S}_{\delta}\right)$ is also a $\delta$-approximate simulation relation of $T_{1}$ by $T_{2}$.

Proof: It is easy to see that we have

$\mathcal{S}_{\delta} \subseteq\left\{\left(q_{1}, q_{2}\right) \in \operatorname{cl}\left(\mathcal{S}_{\delta}\right) \mid \begin{array}{c}\forall q_{1} \stackrel{\sigma}{\rightarrow} 1 q_{1}^{\prime}, \exists q_{2} \stackrel{\sigma}{\rightarrow}{ }_{2} \\ \left(q_{1}^{\prime}, q_{2}^{\prime}\right) \in \operatorname{cl}\left(\mathcal{S}_{\delta}\right)\end{array}, \subseteq \operatorname{cl}\left(\mathcal{S}_{\delta}\right)\right.$.

Then, from Lemma 3, it follows that

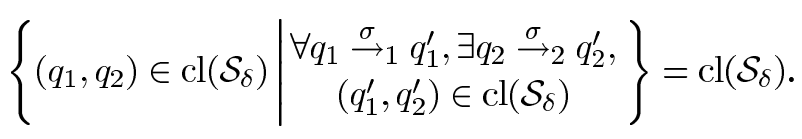

Let $\left(q_{1}, q_{2}\right) \in \operatorname{cl}\left(\mathcal{S}_{\delta}\right)$, there exists a sequence $\left\{\left(q_{1}^{i}, q_{2}^{i}\right)\right\}_{i \in \mathbb{N}}$ of elements of $\mathcal{S}_{\delta}$ converging to $\left(q_{1}, q_{2}\right)$. Since the observation maps $\langle\langle.\rangle\rangle_{1}$ and $\langle\langle.\rangle\rangle_{2}$ are continuous

$$
d_{\Pi}\left(\left\langle\left\langle q_{1}\right\rangle\right\rangle_{1},\left\langle\left\langle q_{2}\right\rangle\right\rangle_{2}\right)=\lim _{i \rightarrow+\infty} d_{\Pi}\left(\left\langle\left\langle q_{1}^{i}\right\rangle\right\rangle_{1},\left\langle\left\langle q_{2}^{i}\right\rangle\right\rangle_{2}\right) \leq \delta .
$$

Together with (2), this allows to conclude that $\operatorname{cl}\left(\mathcal{S}_{\delta}\right)$ is also a $\delta$-approximate simulation relation of $T_{1}$ by $T_{2}$.

Corollary 1: Let $T_{1}, T_{2} \in \mathcal{T}_{M}^{*}(\Sigma, \Pi)$, and let $S_{\delta}^{\max }$ be the maximal $\delta$-approximate simulation relation of $T_{1}$ by $T_{2}$. Then $S_{\delta}^{\max }$ is a closed subset of $Q_{1} \times Q_{2}$.

Proof: $S_{\delta}^{\max }$ is a $\delta$-approximate simulation relation of $T_{1}$ by $T_{2}$, so is $\operatorname{cl}\left(S_{\delta}^{\max }\right)$. Hence, since $S_{\delta}^{\max }$ is the maximal $\delta$-approximate simulation relation of $T_{1}$ by $T_{2}$, we have $\operatorname{cl}\left(S_{\delta}^{\max }\right) \subseteq$ $S_{\delta}^{\max }$.

Before we can state the main result about the simulation metric, we will require the following lemma.

Lemma 4: Let $\left\{\mathcal{R}_{\varepsilon}\right\}_{\varepsilon>0}$ be a family of closed subsets of $Q_{1} \times$ $Q_{2}$ indexed over the strictly positive real numbers and such that for all $\varepsilon_{1} \leq \varepsilon_{2}, \mathcal{R}_{\varepsilon_{1}} \subseteq \mathcal{R}_{\varepsilon_{2}}$. Let $q_{1} \in Q_{1}$ and let $C_{2}$ be a compact subset of $Q_{2}$ :

$$
\begin{aligned}
\forall \varepsilon>0, \exists q_{2} \in C_{2}, \text { such that }\left(q_{1}, q_{2}\right) \in \mathcal{R}_{\varepsilon} \\
\quad \Longrightarrow \exists q_{2} \in C_{2}, \text { such that } \forall \varepsilon>0,\left(q_{1}, q_{2}\right) \in \mathcal{R}_{\varepsilon} .
\end{aligned}
$$

Proof: Let $\left\{\varepsilon_{i}\right\}_{i \in \mathbb{N}}$ be a decreasing sequence of real numbers converging to 0 . Then, for all $i \in \mathbb{N}$, there exists $q_{2}^{i} \in C_{2}$ such that $\left(q_{1}, q_{2}^{i}\right) \in \mathcal{R}_{\varepsilon_{i}}$. Since $C_{2}$ is compact, there exists a subsequence of $\left\{q_{2}^{i}\right\}_{i \in \mathbb{N}}$ which we will also note $\left\{q_{2}^{i}\right\}_{i \in \mathbb{N}}$ and which converges to a limit $q_{2} \in C_{2}$. Let $\varepsilon>0$, there exists $n \in \mathbb{N}$ such that for all $i \geq n, \varepsilon_{i} \leq \varepsilon$ and hence $\mathcal{R}_{\varepsilon_{i}} \subseteq \mathcal{R}_{\varepsilon}$. Therefore, for all $i \geq n,\left(q_{1}, q_{2}^{i}\right) \in \mathcal{R}_{\varepsilon}$ which is closed. Hence, $\left(q_{1}, q_{2}\right) \in \mathcal{R}_{\varepsilon}$.

The main result about simulation metrics states that for regular metric labeled transition systems, the zero section of the simulation metric coincides with the exact simulation relation $\preceq$ of Section II-B. 
Theorem 6: For all $T_{1}, T_{2} \in \mathcal{T}_{M}^{*}(\Sigma, \Pi)$

$$
T_{1} \preceq T_{2} \Longleftrightarrow d_{\mathcal{S}}\left(T_{1}, T_{2}\right)=0 .
$$

Proof: Let $T_{1}, T_{2} \in \mathcal{T}_{M}^{*}(\Sigma, \Pi)$, such that $d_{\mathcal{S}}\left(T_{1}, T_{2}\right)=0$. This implies that for all $\delta>0, T_{1} \preceq_{\delta} T_{2}$. Equivalently, for all $q_{1} \in Q_{1}^{0}$, for all $\delta>0$, there exists $q_{2} \in Q_{2}^{0}$ such that $\left(q_{1}, q_{2}\right) \in \mathcal{S}_{\delta}^{\max }$. From Corollary 1 , for all $\delta>0, \mathcal{S}_{\delta}^{\max }$ is closed. Moreover, since $Q_{2}^{0}$ is compact, it follows from Lemma 4 that for all $q_{1} \in Q_{1}^{0}$, there exists $q_{2} \in Q_{2}^{0}$ such that for all $\delta>$ $0,\left(q_{1}, q_{2}\right) \in \mathcal{S}_{\delta}^{\max }$. Let us define the relation $\mathcal{S}=\bigcap_{\delta>0} \mathcal{S}_{\delta}^{\max }$, we have

$$
\forall q_{1} \in Q_{1}^{0}, \exists q_{2} \in Q_{2}^{0} \text { such that }\left(q_{1}, q_{2}\right) \in \mathcal{S} \text {. }
$$

Let us prove that $\mathcal{S}$ is an exact simulation relation. Let $\left(q_{1}, q_{2}\right) \in$ $\mathcal{S}$,

$$
\begin{aligned}
\forall \delta>0, d_{\Pi}\left(\left\langle\left\langle q_{1}\right\rangle\right\rangle_{1},\left\langle\left\langle q_{2}\right\rangle\right\rangle_{2}\right) \leq \delta \\
\quad \Longleftrightarrow d_{\Pi}\left(\left\langle\left\langle q_{1}\right\rangle\right\rangle_{1},\left\langle\left\langle\left\langle q_{2}\right\rangle\right\rangle_{2}\right)=0\right. \\
\quad \Longleftrightarrow\left\langle\left\langle q_{1}\right\rangle\right\rangle_{1}=\left\langle\left\langle q_{2}\right\rangle\right\rangle_{2} .
\end{aligned}
$$

Let $q_{1} \stackrel{\sigma}{\rightarrow} 1 q_{1}^{\prime}$. For all $\delta>0$, there exists $q_{2} \stackrel{\sigma}{\rightarrow} 2 q_{2}^{\prime}$ such that $\left(q_{1}^{\prime}, q_{2}^{\prime}\right) \in \mathcal{S}_{\delta}^{\max }$. Since $\operatorname{Post}_{2}^{\sigma}\left(q_{2}\right)$ is compact, it follows from Lemma 4 that there exists $q_{2} \stackrel{\sigma}{\rightarrow} 2 q_{2}^{\prime}$ such that for all $\delta>0$, $\left(q_{1}^{\prime}, q_{2}^{\prime}\right) \in \mathcal{S}_{\delta}^{\max }$. Equivalently,

$$
\forall q_{1} \stackrel{\sigma}{\rightarrow}_{1} q_{1}^{\prime}, \exists q_{2} \stackrel{\sigma}{\rightarrow}_{2} q_{2}^{\prime} \text { such that }\left(q_{1}^{\prime}, q_{2}^{\prime}\right) \in \mathcal{S} .
$$

Hence, $\mathcal{S}$ is an exact simulation relation. Equation (3) allows to conclude that $T_{1} \preceq T_{2}$.

The relationship between the simulation metric and the language metric is captured by the following result which holds for all metric transition systems, not necessarily regular.

Lemma 5: For all $T_{1}, T_{2} \in \mathcal{T}_{M}(\Sigma, \Pi), d_{\overrightarrow{\mathcal{L}}}\left(T_{1}, T_{2}\right) \leq$ $d_{\overrightarrow{\mathcal{S}}}\left(T_{1}, T_{2}\right)$.

Proof: Let $\delta>d_{\mathcal{S}}\left(T_{1}, T_{2}\right)$, then $T_{1} \preceq_{\delta} T_{2}$. Let

$$
s_{1}=\left\{\pi_{1}^{i} \stackrel{\sigma_{1}^{i}}{\rightarrow} \pi_{1}^{i+1}\right\}_{i \in \mathbb{N}} \in L\left(T_{1}\right)
$$

there exists a state trajectory of $T_{1}$ :

$$
\left\{q_{1}^{i}{\stackrel{\sigma_{1}^{i}}{\rightarrow}}_{1} q_{1}^{i+1}\right\}_{i \in \mathbb{N}} \text { such that } \forall i \in \mathbb{N},\left\langle\left\langle q_{1}^{i}\right\rangle\right\rangle_{1}=\pi_{1}^{i} .
$$

$q_{1}^{0} \in Q_{1}^{0}$ then there exists $q_{2}^{0} \in Q_{2}^{0}$ such that $\left(q_{1}^{0}, q_{2}^{0}\right)$ is in $\mathcal{S}_{\delta}^{\max }$, the maximal $\delta$-approximate simulation relation of $T_{1}$ by $T_{2}$. Using the second property of Definition 10 it can be shown by induction that there exists a state trajectory of $T_{2}$,

$\left\{q_{2}^{i} \stackrel{\sigma_{2}^{i}}{\rightarrow} 2 q_{2}^{i+1}\right\}_{i \in \mathbb{N}}$ such that

$$
\forall i \in \mathbb{N}, \sigma_{1}^{i}=\sigma_{2}^{i} \text { and }\left(q_{1}^{i}, q_{2}^{i}\right) \in \mathcal{S}_{\delta}^{\max } .
$$

Let $s_{2}=\left\{\pi_{2}^{i \stackrel{\sigma_{2}^{2}}{\rightarrow}} \pi_{2}^{i+1}\right\}_{i \in \mathbb{N}}$ be the associated external trajectory accepted by $T_{2}$ (for all $i \in \mathbb{N},\left\langle\left\langle q_{2}^{i}\right\rangle\right\rangle_{2}=\pi_{2}^{i}$ ). Then, we have for all $i \in \mathbb{N}, d_{\Pi}\left(\pi_{1}^{i}, \pi_{2}^{i}\right)=d_{\Pi}\left(\left\langle\left\langle q_{1}^{i}\right\rangle\right\rangle_{1},\left\langle\left\langle q_{2}^{i}\right\rangle\right\rangle_{2}\right) \leq \delta$. Therefore, since the external trajectories $s_{1}$ and $s_{2}$ share the same sequence of labels, $d_{\mathcal{E}}\left(s_{1}, s_{2}\right) \leq \delta$. Hence, $d_{\mathcal{L}}\left(T_{1}, T_{2}\right) \leq \delta$. This holds for all $\delta>d_{\mathcal{S}}\left(T_{1}, T_{2}\right)$, therefore $d_{\overrightarrow{\mathcal{L}}}\left(T_{1}, T_{2}\right) \leq d_{\overrightarrow{\mathcal{S}}}\left(T_{1}, T_{2}\right)$.

For deterministic transition systems, the equivalence between exact language inclusion and exact simulation has an approximate analogue, as the following result shows. The proof can be found in [34].

Lemma 6: If $T_{2}$ is deterministic then $d_{\overrightarrow{\mathcal{L}}}\left(T_{1}, T_{2}\right)=$ $d_{\overrightarrow{\mathcal{S}}}\left(T_{1}, T_{2}\right)$.

The fact that the simulation metric is stronger (for nondeterministic systems) than the language inclusion metric will result in algorithms for its computation, which are advantageous especially in the context of infinite metric transition systems. Before we discuss their computation in Sections VI and VII, we present similar results for approximate bisimulations.

\section{Approximate Bisimulations and Bisimulation Metric}

The development of approximate bisimulation is similar to the development of approximate simulation. We therefore state all results without their conceptually and technically similar proofs.

1) Approximate Bisimulation: If a relation is a $\delta$-approximate simulation relation of $T_{1}$ by $T_{2}$ as well as a $\delta$-approximate simulation relation of $T_{2}$ by $T_{1}$, then it is called a $\delta$-approximate bisimulation relation.

Definition 14 (Approximate Bisimulation): Let $T_{1}, T_{2} \in$ $\mathcal{T}_{M}(\Sigma, \Pi)$. A relation $\mathcal{B}_{\delta} \subseteq \mathcal{Q}_{1} \times \mathcal{Q}_{2}$ is a $\delta$-approximate bisimulation relation between $T_{1}$ and $T_{2}$ if for all $\left(q_{1}, q_{2}\right) \in \mathcal{B}_{\delta}$

1) $d_{\Pi}\left(\left\langle\left\langle q_{1}\right\rangle\right\rangle_{1},\left\langle\left\langle q_{2}\right\rangle\right\rangle_{2}\right) \leq \delta$

2) $\forall q_{1} \underset{\sigma}{\stackrel{\sigma}{\rightarrow}} 1 q_{1}^{\prime}, \exists q_{2} \underset{\sigma^{\circ}}{\stackrel{\sigma}{\rightarrow}} q_{2}^{\prime}$ such that $\left(q_{1}^{\prime}, q_{2}^{\prime}\right) \in \mathcal{B}_{\delta}$

3) $\forall q_{2} \stackrel{\sigma}{\rightarrow}_{2} q_{2}^{\prime}, \exists q_{1} \stackrel{\sigma}{\rightarrow}_{1} q_{1}^{\prime}$ such that $\left(q_{1}^{\prime}, q_{2}^{\prime}\right) \in \mathcal{B}_{\delta}$.

Definition 15: $T_{1}$ and $T_{2}$ are said to be approximately bisimilar with the precision $\delta$ (denoted $T_{1} \cong_{\delta} T_{2}$ ), if there exists $\mathcal{B}_{\delta}$, a $\delta$-approximate bisimulation relation between $T_{1}$ and $T_{2}$ such that for all $q_{1} \in \mathcal{Q}_{1}^{0}$, there exists $q_{2} \in \mathcal{Q}_{2}^{0}$ such that $\left(q_{1}, q_{2}\right) \in \mathcal{B}_{\delta}$, and conversely.

Similar to approximate simulation relations, we can show that the union of a (possibly uncountable) family of $\delta$-approximate bisimulation relations between $T_{1}$ and $T_{2}$ is a $\delta$-approximate bisimulation relation between $T_{1}$ and $T_{2}$. It follows that there exists a maximal $\delta$-approximate bisimulation relation between $T_{1}$ and $T_{2}$.

Definition 16: Let $\left\{\mathcal{B}_{\delta}^{i}\right\}_{i \in I}$ be the set of $\delta$-approximate bisimulation relations between $T_{1}$ and $T_{2}$. The maximal $\delta$-approximate bisimulation relation between $T_{1}$ and $T_{2}$ is defined by

$$
\mathcal{B}_{\delta}^{\max }=\bigcup_{i \in I} \mathcal{B}_{\delta}^{i}
$$

Clearly, $T_{1}$ and $T_{2}$ are approximately bisimilar with precision $\delta$ if and only if for all $q_{1} \in Q_{1}^{0}$, there exists $q_{2} \in Q_{2}^{0}$ such 
that $\left(q_{1}, q_{2}\right) \in \mathcal{B}_{\delta}^{\max }$, and conversely. Approximate bisimulation relations for metric transition systems satisfy the following properties.

Proposition 4: Let $T_{1}, T_{2}$ and $T_{3} \in \mathcal{T}_{M}(\Sigma, \Pi)$.

1) For all $\delta \geq 0, T_{1} \cong_{\delta} T_{1}$.

2) For all $\delta \geq 0$, if $T_{1} \cong_{\delta} T_{2}$, then for all $\delta^{\prime} \geq \delta, T_{1} \cong_{\delta}^{\prime} T_{2}$.

3) For all $\delta \geq 0, \delta^{\prime} \geq 0$, if $T_{1} \cong_{\delta} T_{2}$ and $T_{2} \cong_{\delta^{\prime}} T_{3}$, then $T_{1} \cong_{\delta+\delta^{\prime}} T_{3}$.

Contrarily to $\cong$, the relation $\cong_{\delta}$ (for $\delta>0$ ) is not an equivalence relation ${ }^{3}$ on the set of metric labeled transition systems $\mathcal{T}_{M}(\Sigma, \Pi)$. But the above properties enable us to define a bisimulation metric in $\mathcal{T}_{M}(\Sigma, \Pi)$.

2) Bisimulation Metric:

Definition 17 (Bisimulation Metric): The bisimulation metric is the function defined by

$$
d_{\mathcal{B}}\left(T_{1}, T_{2}\right)=\inf \left\{\delta \mid T_{1} \cong_{\delta} T_{2}\right\}
$$

Theorem 7: The bisimulation metric is a pseudo metric on the set of metric transition systems $\mathcal{T}_{M}(\Sigma, \Pi)$ and

$$
T_{1} \cong T_{2} \Longrightarrow d_{\mathcal{B}}\left(T_{1}, T_{2}\right)=0 .
$$

Lemma 7: For all $T_{1}, T_{2} \in \mathcal{T}_{M}(\Sigma, \Pi), d_{\mathcal{L}}\left(T_{1}, T_{2}\right) \leq$ $d_{\mathcal{B}}\left(T_{1}, T_{2}\right)$ and $d_{\mathcal{S}}\left(T_{1}, T_{2}\right) \leq d_{\mathcal{B}}\left(T_{1}, T_{2}\right)$.

Proof: The proof of the first inequality is similar to the proof of Lemma 5. Let us remark that a $\delta$-approximate bisimulation relation is also a $\delta$-approximate simulation relation. Hence, $T_{1} \cong_{\delta} T_{2}$ implies that $T_{1} \preceq_{\delta} T_{2}$ and therefore $d_{\mathcal{S}}\left(T_{1}, T_{2}\right) \leq$ $d_{\mathcal{B}}\left(T_{1}, T_{2}\right)$.

If we assume that the metric transition systems we consider are regular, then, similar to the simulation metric, we obtain that the zero section of the bisimulation metric coincides with the exact equivalence relation $\cong$ from Section II-B.

Theorem 8: For all $T_{1}, T_{2} \in \mathcal{T}_{M}^{*}(\Sigma, \Pi)$,

$$
T_{1} \cong T_{2} \Longleftrightarrow d_{\mathcal{B}}\left(T_{1}, T_{2}\right)=0
$$

For deterministic systems, the notions of language equivalence and exact bisimulation holds also between the approximate versions of these notions. It implies that for deterministic systems the language and the bisimulation metrics are equal.

Lemma 8: If $T_{1}$ and $T_{2}$ are deterministic then $d_{\mathcal{L}}\left(T_{1}, T_{2}\right)=$ $d_{\mathcal{B}}\left(T_{1}, T_{2}\right)$.

\footnotetext{
${ }^{3}$ However, the relation $T_{1} \approx T_{2}$ defined as $\exists \delta: T_{1} \cong{ }_{\delta} T_{2}$ is an equivalence
} relation in $\mathcal{T}_{M}(\Sigma, \Pi)$.

\section{Hierarchy of System Approximations}

The results of Lemmas 1, 5, and 7 can be summarized in the following theorem which is the analogue of Theorem 1 for our approximation metrics.

Theorem 9 (Hierarchy of System Approximations): For all metric transition system $T_{1}, T_{2} \in \mathcal{T}_{M}(\Sigma, \Pi)$, the following relationships hold (where $\rightarrow$ stands for $\geq$ )

$$
\begin{array}{ccccc}
d_{\mathcal{B}}\left(T_{1}, T_{2}\right) & \rightarrow & d_{\mathcal{L}}\left(T_{1}, T_{2}\right) & \rightarrow & d_{\mathcal{R}}\left(T_{1}, T_{2}\right) \\
\downarrow & & \downarrow & & \downarrow \\
d_{\overrightarrow{\mathcal{S}}}\left(T_{1}, T_{2}\right) & \rightarrow & d_{\overrightarrow{\mathcal{L}}}\left(T_{1}, T_{2}\right) & \rightarrow & d_{\overrightarrow{\mathcal{R}}}\left(T_{1}, T_{2}\right) .
\end{array}
$$

All the metrics defined in this section provide an over-approximation of the directed reachability metric which is useful for reducing the complexity of the safety verification problem (see (1)). Let us remark that for regular metric labeled transition systems, a slightly weaker version of Theorem 1 is obtained by considering the zero sections of the different metrics, as shown in the equation at the bottom of the page.

For deterministic labeled transition systems, according to Lemmas 6 and 8, some of the approximation metrics are equal. The following theorem summarizes these results.

Theorem 10: If $T_{1}$ and $T_{2}$ are deterministic then the following equalities hold:

$$
\begin{aligned}
d_{\mathcal{B}}\left(T_{1}, T_{2}\right) & =d_{\mathcal{L}}\left(T_{1}, T_{2}\right) \\
d_{\overrightarrow{\mathcal{S}}}\left(T_{1}, T_{2}\right) & =d_{\overrightarrow{\mathcal{L}}}\left(T_{1}, T_{2}\right) .
\end{aligned}
$$

\section{COMPOSITIONAL APPROXIMATIONS}

One of the most powerful features of simulation and bisimulation is that they allow compositional reasoning. In fact, simulation and bisimulation have their origins in concurrency theory [1], before impacting formal verification [2]. In this section, we show that the approximate metrics we developed in the previous section are also compositional, in an approximate sense. Because of the lack of space, the proofs of this section are omitted but can be found in [34]. We illustrate the compositionality of our metrics for a synchronous composition operator. We define the composition of two metric transition systems $T_{1}=\left(Q_{1}, \Sigma_{1}, \rightarrow_{1}, Q_{1}^{0}, \Pi_{1},\langle\langle.\rangle\rangle_{1}\right)$ and $T_{2}=\left(Q_{2}, \Sigma_{2}, \rightarrow_{2}, Q_{2}^{0}, \Pi_{2},\langle\langle\cdot\rangle\rangle_{2}\right)$ is denoted $T_{1} \| T_{2}$ and is defined by $T_{1} \| T_{2}=\left(Q, \Sigma, \rightarrow, Q^{0}, \Pi,\langle\langle\cdot\rangle\rangle\right)$ where

- the set of states $Q=Q_{1} \times Q_{2}$;

- the set of labels $\Sigma=\Sigma_{1} \cap \Sigma_{2}$;

- the transition relation is given by

$$
\left(q_{1}, q_{2}\right) \stackrel{\sigma}{\rightarrow}\left(q_{1}^{\prime}, q_{2}^{\prime}\right) \Longleftrightarrow q_{1} \stackrel{\sigma}{\rightarrow}_{1} q_{1}^{\prime} \text { and } q_{2} \stackrel{\sigma}{\rightarrow}_{2} q_{2}^{\prime} ;
$$

$$
\begin{gathered}
T_{1} \cong T_{2} \Rightarrow \operatorname{cl}\left(L\left(T_{1}\right)\right)=\operatorname{cl}\left(L\left(T_{2}\right)\right) \Rightarrow \operatorname{cl}\left(\operatorname{Reach}\left(T_{1}\right)\right)=\operatorname{cl}\left(\operatorname{Reach}\left(T_{2}\right)\right) \\
\underset{\Downarrow}{\Downarrow} \\
T_{1} \preceq T_{2} \Rightarrow \operatorname{cl}\left(L\left(T_{1}\right)\right) \subseteq \operatorname{cl}\left(L\left(T_{2}\right)\right) \Rightarrow \operatorname{cl}\left(\operatorname{Reach}\left(T_{1}\right)\right) \subseteq \operatorname{cl}\left(\operatorname{Reach}\left(T_{2}\right)\right)
\end{gathered}
$$


- the set of initial states $Q^{0}=Q_{1}^{0} \times Q_{2}^{0}$;

- the set of observations $\Pi=\Pi_{1} \times \Pi_{2}$;

- the observation map is given by $\left\langle\left\langle\left(q_{1}, q_{2}\right)\right\rangle\right\rangle=$ $\left(\left\langle\left\langle q_{1}\right\rangle\right\rangle_{1},\left\langle\left\langle q_{2}\right\rangle\right\rangle_{2}\right)$.

Therefore, both systems are observed and synchronize on common events. ${ }^{4}$ We assume that the composition is non-blocking. Since $\left(\Pi_{1}, d_{\Pi_{1}}\right)$ and $\left(\Pi_{2}, d_{\Pi_{2}}\right)$ are metric spaces, we consider the metric space $\left(\Pi, d_{\Pi}\right)$ where the metric $d_{\Pi}$ is defined by

$$
d_{\Pi}\left(\left(\pi_{1}, \pi_{2}\right),\left(\pi_{1}^{\prime}, \pi_{2}^{\prime}\right)\right)=d_{\Pi_{1}}\left(\pi_{1}, \pi_{1}^{\prime}\right)+d_{\Pi_{2}}\left(\pi_{2}, \pi_{2}^{\prime}\right) .
$$

If $U_{1}=\left(P_{1}, \Sigma_{1}, \rightarrow_{1}, P_{1}^{0}, \Pi_{1},\langle\langle.\rangle\rangle_{1}\right)$ is an approximation of $T_{1}$, and $U_{2}=\left(P_{2}, \Sigma_{2}, \rightarrow_{2}, P_{2}^{0}, \Pi_{2},\langle\langle.\rangle\rangle_{2}\right)$ is an approximation of $T_{2}$, we show that $U_{1} \| U_{2}$ is an approximation of $T_{1} \| T_{2}$, from the perspective of our language metrics.

Theorem 11: For all $T_{1}, U_{1} \in \mathcal{T}_{M}\left(\Sigma_{1}, \Pi_{1}\right), T_{2}, U_{2} \in$ $\mathcal{T}_{M}\left(\Sigma_{2}, \Pi_{2}\right)$

$$
\begin{aligned}
d_{\mathcal{L}}\left(T_{1}\left\|T_{2}, U_{1}\right\| U_{2}\right) & \leq d_{\mathcal{L}}\left(T_{1}, U_{1}\right)+d_{\overrightarrow{\mathcal{L}}}\left(T_{2}, U_{2}\right) \\
d_{\mathcal{L}}\left(T_{1}\left\|T_{2}, U_{1}\right\| U_{2}\right) & \leq d_{\mathcal{L}}\left(T_{1}, U_{1}\right)+d_{\mathcal{L}}\left(T_{2}, U_{2}\right) .
\end{aligned}
$$

Therefore, approximate language inclusion is compositional. The following results show that it is also the case for approximate simulation and approximate bisimulation.

Proposition 5: Let $T_{1}, U_{1} \in \mathcal{T}_{M}\left(\Sigma_{1}, \Pi_{1}\right), T_{2}, U_{2} \in$ $\mathcal{T}_{M}\left(\Sigma_{2}, \Pi_{2}\right)$, then

$$
\begin{aligned}
& T_{1} \preceq_{\delta_{1}} U_{1} \text { and } T_{2} \preceq_{\delta_{2}} U_{2} \quad \Longrightarrow \quad T_{1}\left\|T_{2} \preceq_{\delta_{1}+\delta_{2}} U_{1}\right\| U_{2} \\
& T_{1} \cong_{\delta_{1}} U_{1} \text { and } T_{2} \cong_{\delta_{2}} U_{2} \quad \Longrightarrow \quad T_{1}\left\|T_{2} \cong_{\delta_{1}+\delta_{2}} U_{1}\right\| U_{2} .
\end{aligned}
$$

As a consequence, we have the following Theorem.

Theorem 12: For all $T_{1}, U_{1} \in \mathcal{T}_{M}\left(\Sigma_{1}, \Pi_{1}\right), T_{2}, U_{2} \in$ $\mathcal{T}_{M}\left(\Sigma_{2}, \Pi_{2}\right)$

$$
\begin{aligned}
d_{\mathcal{S}}\left(T_{1}\left\|T_{2}, U_{1}\right\| U_{2}\right) & \leq d_{\mathcal{S}}\left(T_{1}, U_{1}\right)+d_{\mathcal{S}}\left(T_{2}, U_{2}\right) \\
d_{\mathcal{B}}\left(T_{1}\left\|T_{2}, U_{1}\right\| U_{2}\right) & \leq d_{\mathcal{B}}\left(T_{1}, U_{1}\right)+d_{\mathcal{B}}\left(T_{2}, U_{2}\right)
\end{aligned}
$$

In this part, we showed that our approximation framework allows compositional reasoning. Indeed, the composition of approximations is an approximation of the composition. Note that even though our compositionality results hold for the language, simulation, and bisimulation metric, they do not hold for the reachability metric. This is further evidence that for safety verification, overapproximating the reachability metric with the language, simulation, or bisimulation metric, can further decompose safety analysis by exploiting the above compositionality results.

\section{EXACT METRIC COMPUTATION}

In the previous sections, we presented a compositional theory of system approximation for metric transition systems. In this section, we focus on the computation of the simulation and bisimulation metrics since the language (and hence reachability) metrics are either impossible to compute for infinite transition systems, or computationally demanding for finite quantitative transition systems [16].

\footnotetext{
${ }^{4}$ More general composition operators can and will be considered in future work.
}

We propose two approaches for computing the simulation and bisimulation metric. The first approach, described in this Section, focuses on computing exactly the metrics using a natural generalization of the fixed-point (or game-theoretic) interpretations of simulation and bisimulation. The second approach, described in Section VII, is a relaxation of the first approach, offering approximate upper bounds for the metrics at a reduced computational cost.

\section{A. Maximal Approximate Simulations}

For the established exact simulations of Section II-B, a computable characterization of the maximal exact simulation relation is often given in terms of the fixed point of a decreasing sequence of subsets of $\mathcal{Q}_{1} \times \mathcal{Q}_{2}$. A similar approach can be used for the maximal $\delta$-approximate simulation relation. We assume that the metric transition systems we consider are regular. Let us consider the following algorithm whose goal is to search for such relations.

Algorithm 1: Let $T_{1}, T_{2} \in \mathcal{T}_{M}^{*}(\Sigma, \Pi)$. For a given $\delta \geq 0$, define the following sequence $\left\{\mathcal{S}_{\delta}^{i}\right\}_{i \in \mathbb{N}}$ of subsets of $\mathcal{Q}_{1} \times \mathcal{Q}_{2}$ :

$$
\begin{aligned}
\mathcal{S}_{\delta}^{0} & =\left\{\left(q_{1}, q_{2}\right) \in \mathcal{Q}_{1} \times \mathcal{Q}_{2} \mid d_{\Pi}\left(\left\langle\left\langle q_{1}\right\rangle\right\rangle_{1},\left\langle\left\langle q_{2}\right\rangle\right\rangle_{2}\right) \leq \delta\right\} \\
\mathcal{S}_{\delta}^{i+1} & =\left\{\begin{array}{l|l}
\left(q_{1}, q_{2}\right) \in \mathcal{S}_{\delta}^{i} & \begin{array}{l}
\forall q_{1} \stackrel{\sigma}{\rightarrow} q_{1}^{\prime}, \exists q_{2} \stackrel{\sigma}{\rightarrow} q_{2}^{\prime}, \\
\text { such that }\left(q_{1}^{\prime}, q_{2}^{\prime}\right) \in \mathcal{S}_{\delta}^{i}
\end{array}
\end{array}\right\} .
\end{aligned}
$$

Lemma 9: For all $\delta \geq 0$, for all $i \in \mathbb{N}$, the subset $\mathcal{S}_{\delta}^{i}$ is closed.

Proof: Since the observation maps $\langle\langle\cdot\rangle\rangle_{1}$ and $\langle\langle.\rangle\rangle_{2}$ are continuous, it is clear that the subset $\mathcal{S}_{\delta}^{0}$ is closed. Assuming that the subset $\mathcal{S}_{\delta}^{i}$ is closed for some $i \in \mathbb{N}$, then, according to Lemma $3, \mathcal{S}_{\delta}^{i+1}$ is closed as well.

For metric transition systems with a finite number of states, it is clear that Algorithm 1 reaches a fixed point in a finite number of steps. For infinite transition systems, Algorithm 1 may not reach a fixed point in a finite number steps. However, the sequence $\left\{\mathcal{S}_{\delta}^{i}\right\}_{i \in \mathbb{N}}$ does approach a fixed point as $i$ goes to $+\infty$. This fixed point is the maximal $\delta$-approximate simulation relation of $T_{1}$ by $T_{2}$.

Theorem 13: Let $\left\{\mathcal{S}_{\delta}^{i}\right\}_{i \in \mathbb{N}}$ be the decreasing sequence of sets defined by Algorithm 1 and $\mathcal{S}_{\delta}^{\max }$ be the maximal $\delta$-approximate simulation relation of $T_{1}$ by $T_{2}$. Then, the following properties hold:

$$
\forall i \in \mathbb{N}, \mathcal{S}_{\delta}^{\max } \subseteq \mathcal{S}_{\delta}^{i} \bigcap_{i=0}^{i=+\infty} \mathcal{S}_{\delta}^{i}=\mathcal{S}_{\delta}^{\max } .
$$

Proof: It is clear that $\mathcal{S}_{\delta}^{\max } \subseteq \mathcal{S}_{\delta}^{0}$. Hence, let us assume that $\mathcal{S}_{\delta}^{\max } \subseteq \mathcal{S}_{\delta}^{i}$, for some $i \in \mathbb{N}$. Let $\left(q_{1}, q_{2}\right) \in \mathcal{S}_{\delta}^{\max } \subseteq \mathcal{S}_{\delta}^{i}$, for all $q_{1} \stackrel{\sigma}{\rightarrow} 1$ q $q_{1}^{\prime}$, there exists $q_{2} \stackrel{\sigma^{\prime}}{\rightarrow} \quad q_{2}^{\prime}$ such that $\left(q_{1}^{\prime}, q_{2}^{\prime}\right) \in \mathcal{S}_{\delta}^{\max } \subseteq \mathcal{S}_{\delta}^{i}$. Hence, $\left(q_{1}, q_{2}\right) \in \mathcal{S}_{\delta}^{i+1}$. By induction, the first part of the theorem is proved. Now, let us show that $\bigcap_{i=0}^{i=+\infty} \mathcal{S}_{\delta}^{i}$ is a $\delta$-approximate simulation relation of $T_{1}$ by $T_{2}$. Let $\left(q_{1}, q_{2}\right) \in \bigcap_{i=0}^{i=+\infty} \mathcal{S}_{\delta}^{i}$, then particularly $\left(q_{1}, q_{2}\right) \in \mathcal{S}_{\delta}^{0}$. Hence, $d_{\Pi}\left(\left\langle\left\langle q_{1}\right\rangle\right\rangle_{1},\left\langle\left\langle q_{2}\right\rangle\right\rangle_{2}\right) \leq \delta$. Let $q_{1} \stackrel{\sigma}{\rightarrow} q_{1}^{\prime}$, from the construction of the sequence $\left\{\mathcal{S}_{\delta}^{i}\right\}_{i \in \mathbb{N}}$, for all $i \in \mathbb{N}$, there exists $q_{2}^{\prime i} \in \operatorname{Post}_{2}^{\sigma}\left(q_{2}\right)$ such that $\left(q_{1}^{\prime}, q_{2}^{\prime i}\right) \in \mathcal{S}_{\delta}^{i}$. Since Post ${ }_{2}^{\sigma}\left(q_{2}\right)$ is compact, there exists $\left\{q_{2}^{\prime i_{k}}\right\}_{k \in \mathbb{N}}$ a subsequence of $\left\{q_{2}^{i i}\right\}_{i \in \mathbb{N}}$ converging to an element $q_{2}^{\prime}$ in Post $_{2}^{\sigma}\left(q_{2}\right)$. Let $i \in N$, there exists $n \in \mathbb{N}$ such that for all $k \geq n, i_{k} \geq i$ and, hence, 
$\mathcal{S}_{\delta}^{i_{k}} \subseteq \mathcal{S}_{\delta}^{i}$ because the sequence $\left\{\mathcal{S}_{\delta}^{i}\right\}_{i \in \mathbb{N}}$ is decreasing. Thus, for all $k \geq n,\left(q_{1}^{\prime}, q_{2}^{\prime i_{k}}\right)$ is an element of $\mathcal{S}_{\delta}^{i}$ which is closed. Hence, $\left(q_{1}^{\prime}, q_{2}^{\prime}\right)$ is in $\mathcal{S}_{\delta}^{i}$ for all $i \in \mathbb{N}$. It follows that $\bigcap_{i=0}^{i=+\infty} \mathcal{S}_{\delta}^{i}$ is a $\delta$-approximate simulation of $T_{1}$ by $T_{2}$. From the first part of the theorem, it is clear that $\mathcal{S}_{\delta}^{\max } \subseteq \bigcap_{i=0}^{i=+\infty} \mathcal{S}_{\delta}^{i}$ which allows to conclude.

\section{B. Directed Branching Distance}

A dual approach to Algorithm 1 consists in characterizing the maximal approximate simulation relations of $T_{1}$ by $T_{2}$ as the level sets of a function. Let us consider the following algorithm.

Algorithm 2: Let $T_{1}, T_{2} \in \mathcal{T}_{M}^{*}(\Sigma, \Pi)$. Define the following sequence $\left\{f_{\mathcal{S}}^{i}\right\}_{i \in \mathbb{N}}$ of functions from $\mathcal{Q}_{1} \times \mathcal{Q}_{2}$ to $\mathbb{R}^{+} \cup\{+\infty\}$ :

$$
\begin{aligned}
& f_{\mathcal{S}}^{0}\left(q_{1}, q_{2}\right)=d_{\Pi}\left(\left\langle\left\langle q_{1}\right\rangle\right\rangle_{1},\left\langle\left\langle q_{2}\right\rangle\right\rangle_{2}\right) \\
& f_{\mathcal{S}}^{i+1}\left(q_{1}, q_{2}\right)=\max \left(d_{\Pi}\left(\left\langle\left\langle q_{1}\right\rangle\right\rangle_{1},\left\langle\left\langle q_{2}\right\rangle\right\rangle_{2}\right),\right. \\
&\left.\sup _{q_{1} \stackrel{\sigma}{\rightarrow} q_{1}^{\prime} q_{q_{2}}{ }_{q_{2}} q_{2}^{\prime}} f_{\mathcal{S}}^{i}\left(q_{1}^{\prime}, q_{2}^{\prime}\right)\right), i \in \mathbb{N} .
\end{aligned}
$$

For transition systems with a finite number of states, it is shown in [16], that Algorithm 2 reaches a fixed point in a finite (polynomial) number of steps. In the more general case of metric transition systems, the following lemma shows that the sequence of functions $\left\{f_{\mathcal{S}}^{i}\right\}_{i \in \mathbb{N}}$ converges in a pointwise sense.

Lemma 10: Let $\left\{f_{\mathcal{S}}^{i}\right\}_{i \in \mathbb{N}}$ be the sequence of functions defined by Algorithm 2. For all $\left(q_{1}, q_{2}\right) \in Q_{1} \times Q_{2}$, the sequence $\left\{f_{\mathcal{S}}^{i}\left(q_{1}, q_{2}\right)\right\}_{i \in \mathbb{N}}$ is increasing.

Proof: For all $\left(q_{1}, q_{2}\right) \in Q_{1} \times Q_{2}$, it is clear that $f_{\mathcal{S}}^{0}\left(q_{1}, q_{2}\right) \leq f_{\mathcal{S}}^{1}\left(q_{1}, q_{2}\right)$. Let us assume that for some $i \in \mathbb{N}$, for all $\left(q_{1}, \bar{q}_{2}\right) \in Q_{1} \times Q_{2}, f_{\mathcal{S}}^{i}\left(q_{1}, q_{2}\right) \leq f_{\mathcal{S}}^{i+1}\left(q_{1}, q_{2}\right)$. Let $\left(q_{1}, q_{2}\right) \in Q_{1} \times Q_{2}$, then it is clear that

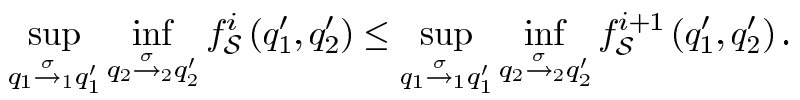

Hence, $f_{\mathcal{S}}^{i+1}\left(q_{1}, q_{2}\right) \leq f_{\mathcal{S}}^{i+2}\left(q_{1}, q_{2}\right)$.

As a consequence of Lemma 10 , for all $\left(q_{1}, q_{2}\right) \in Q_{1} \times Q_{2}$, the sequence $\left\{f_{\mathcal{S}}^{i}\left(q_{1}, q_{2}\right)\right\}_{i \in \mathbb{N}}$ converges in $\mathbb{R}^{+} \cup\{+\infty\}$. Hence, the sequence of functions $\left\{f_{\mathcal{S}}^{i}\right\}_{i \in \mathbb{N}}$ converges pointwise to a limit introduced in [16] for transition systems with a finite set of states as the branching distance.

Definition 18: Let $\left\{f_{\mathcal{S}}^{i}\right\}_{i \in \mathbb{N}}$ be the sequence of functions defined by Algorithm 2. The directed branching distance [16] between $T_{1}$ and $T_{2}$ is the function defined by

$$
\forall\left(q_{1}, q_{2}\right) \in Q_{1} \times Q_{2}, f_{\mathcal{S}}^{\min }\left(q_{1}, q_{2}\right)=\lim _{i \rightarrow \infty} f_{\mathcal{S}}^{i}\left(q_{1}, q_{2}\right)
$$

Before giving the main result on the duality between the approach using relations and the approach using functions, we will need the following lemma.

Lemma 11: Let $f: Q_{1} \times Q_{2}: \rightarrow \mathbb{R}^{+} \cup\{+\infty\}$ be a function with closed level sets: For all $\delta \geq 0$,

$$
\mathcal{V}_{\delta}(f)=\left\{\left(q_{1}, q_{2}\right) \in Q_{1} \times Q_{2} \mid f\left(q_{1}, q_{2}\right) \leq \delta\right\}
$$

is a closed subset. Let $\delta \geq 0, q_{1} \in Q_{1}$ and let $C_{2}$ be a compact subset of $Q_{2}$,

$\forall \varepsilon>0, \exists q_{2} \in C_{2}$, such that $f\left(q_{1}, q_{2}\right) \leq \delta+\varepsilon$

$$
\Longrightarrow \exists q_{2} \in C_{2} \text {, such that } f\left(q_{1}, q_{2}\right) \leq \delta \text {. }
$$

Proof: Let us remark that the family of sets $\left\{\mathcal{V}_{\delta+\varepsilon}(f)\right\}_{\varepsilon>0}$ satisfies the assumptions of Lemma 4. Hence, if for all $\varepsilon>0$ there exists $q_{2} \in C_{2}$ such that $f\left(q_{1}, q_{2}\right) \leq \delta+\varepsilon$ (i.e., $\left(q_{1}, q_{2}\right) \in$ $\left.\mathcal{V}_{\delta+\varepsilon}(f)\right)$, then from Lemma 4 , there exists $q_{2} \in C_{2}$ such that for all $\varepsilon>0,\left(q_{1}, q_{2}\right) \in \mathcal{V}_{\delta+\varepsilon}(f)$ (i.e., $\left.f\left(q_{1}, q_{2}\right) \leq \delta+\varepsilon\right)$. Since this holds for all $\varepsilon>0$, it follows that $f\left(q_{1}, q_{2}\right) \leq \delta$.

Theorem 14: Let $\left\{\mathcal{S}_{\delta}^{i}\right\}_{i \in \mathbb{N}}$ be the sequence of sets defined by Algorithm 1 and $\left\{f_{\mathcal{S}}^{i}\right\}_{i \in \mathbb{N}}$ be the sequence of functions defined by Algorithm 2. Then, for all $i \in N$,

$$
\forall \delta \geq 0, \mathcal{S}_{\delta}^{i}=\left\{\left(q_{1}, q_{2}\right) \in Q_{1} \times Q_{2} \mid f_{\mathcal{S}}^{i}\left(q_{1}, q_{2}\right) \leq \delta\right\} .
$$

Let $\mathcal{S}_{\delta}^{\max }$ be the maximal $\delta$-approximate simulation relation of $T_{1}$ by $T_{2}$ and $f_{\mathcal{S}}^{\min }$ be the directed branching distance between $T_{1}$ and $T_{2}$. Then

$$
\forall \delta \geq 0, \mathcal{S}_{\delta}^{\max }=\left\{\left(q_{1}, q_{2}\right) \in Q_{1} \times Q_{2} \mid f_{\mathcal{S}}^{\min }\left(q_{1}, q_{2}\right) \leq \delta\right\} .
$$

Proof: Let us prove the first part of the theorem. For $i=0$, it is clear that (4) holds. Let us assume that (4) holds, for some $i \in \mathbb{N}$. Let $\delta \geq 0$, let $\left(q_{1}, q_{2}\right) \in \mathcal{S}_{\delta}^{i+1}$, then for all $q_{1} \stackrel{\sigma}{\rightarrow}{ }_{1} q_{1}^{\prime}$, there exists $q_{2} \stackrel{\sigma}{\rightarrow}_{2} q_{2}^{\prime}$ such that $\left(q_{1}^{\prime}, q_{2}^{\prime}\right) \in \mathcal{S}_{\delta}^{i}\left(\right.$ i.e. $f_{\mathcal{S}}^{i}\left(q_{1}^{\prime}, q_{2}^{\prime}\right) \leq$ $\delta$ ). Therefore, we have

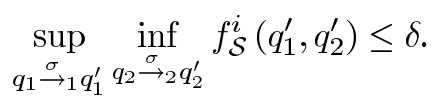

In addition, since $\left(q_{1}, q_{2}\right) \in \mathcal{S}_{\delta}^{i+1} \subseteq \mathcal{S}_{\delta}^{i}$, we have $d_{\Pi}\left(\left\langle\left\langle q_{1}\right\rangle\right\rangle_{1},\left\langle\left\langle q_{2}\right\rangle\right\rangle_{2}\right) \leq f_{\mathcal{S}}^{i}\left(q_{1}, q_{2}\right) \leq \delta$. Hence, $f_{\mathcal{S}}^{i+1}\left(q_{1}, q_{2}\right) \leq$ $\delta$. Reciprocally, let $\left(q_{1}, q_{2}\right)$ be an element of $Q_{1} \times Q_{2}$, such that $f_{\mathcal{S}}^{i+1}\left(q_{1}, q_{2}\right) \leq \delta$. Let $q_{1} \stackrel{\sigma}{\rightarrow}_{1} q_{1}^{\prime}$, then for all $\varepsilon>0$, there exists $q_{2}^{\prime} \in \operatorname{Post}_{2}^{\sigma}\left(q_{2}\right)$, such that $f_{\mathcal{S}}^{i}\left(q_{1}^{\prime}, q_{2}^{\prime}\right) \leq \delta+\varepsilon$. From Lemma 9 , for all $\varepsilon>0, \mathcal{S}_{\delta+\varepsilon}^{i}$ is a closed subset, hence $f_{\mathcal{S}}^{i}$ has closed level sets. It follows from Lemma 11 that there exists $q_{2}^{\prime} \in \operatorname{Post}_{2}^{\sigma}\left(q_{2}\right)$ such that $f_{\mathcal{S}}^{i}\left(q_{1}^{\prime}, q_{2}^{\prime}\right) \leq \delta$ (i.e. $\left.\left(q_{1}^{\prime}, q_{2}^{\prime}\right) \in \mathcal{S}_{\delta}^{i}\right)$. Now let us remark that $f_{\mathcal{S}}^{i}\left(q_{1}, q_{2}\right) \leq f_{\mathcal{S}}^{i+1}\left(q_{1}, q_{2}\right) \leq \delta$, hence $\left(q_{1}, q_{2}\right) \in \mathcal{S}_{\delta}^{i}$. Therefore, $\left(q_{1}, q_{2}\right) \in \mathcal{S}_{\delta}^{i+1}$. Hence, the first part of the theorem is proved by induction. The second part of the theorem is straightforward from the following sequence of equivalences:

$$
\begin{aligned}
f_{\mathcal{S}}^{\min }\left(q_{1}, q_{2}\right) \leq \delta & \Longleftrightarrow \forall i \in \mathbb{N}, f_{\mathcal{S}}^{i}\left(q_{1}, q_{2}\right) \leq \delta \\
& \Longleftrightarrow \forall i \in \mathbb{N},\left(q_{1}, q_{2}\right) \in \mathcal{S}_{\delta}^{i} \\
& \Longleftrightarrow\left(q_{1}, q_{2}\right) \in \mathcal{S}_{\delta}^{\max } .
\end{aligned}
$$

Let us remark that particularly, the zero set of the directed branching distance between $T_{1}$ and $T_{2}$ is the maximal exact simulation relation of $T_{1}$ by $T_{2}$. Another interesting fact is that the level sets of the functions $\left\{f_{\mathcal{S}}^{i}\right\}_{i \in \mathbb{N}}$ and $f_{\mathcal{S}}^{\min }$ are closed subsets.

For metric transition systems with an infinite set of states, the fixed point iteration of Algorithm 2 may not be an efficient 
way to compute the directed branching distance. An alternative method is to solve the following fixed-point equation.

Theorem 15: The directed branching distance between $T_{1}$ and $T_{2}$ is the smallest function defined on $Q_{1} \times Q_{2}$ with values in $\mathbb{R}^{+} \cup\{+\infty\}$ satisfying the following functional equation:

$$
\begin{aligned}
& f_{\mathcal{S}}^{\min }\left(q_{1}, q_{2}\right)=\max \left(d_{\Pi}\left(\left\langle\left\langle q_{1}\right\rangle\right\rangle_{1},\left\langle\left\langle q_{2}\right\rangle\right\rangle_{2}\right),\right.
\end{aligned}
$$

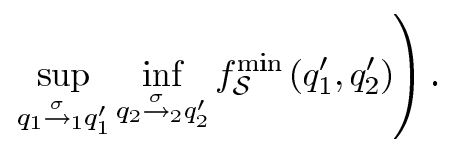

Proof: Let $\left(q_{1}, q_{2}\right) \in Q_{1} \times Q_{2}$, for all $i \in \mathbb{N}$, we have $f_{\mathcal{S}}^{i}\left(q_{1}, q_{2}\right) \leq f_{\mathcal{S}}^{\min }\left(q_{1}, q_{2}\right)$. Hence, for all $i \in \mathbb{N}$

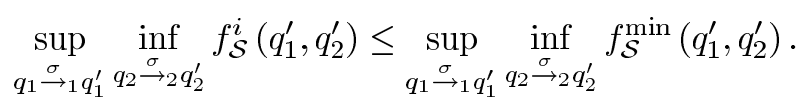

Therefore, for all $i \in \mathbb{N}$, we have

$$
\begin{aligned}
& f_{\mathcal{S}}^{i+1}\left(q_{1}, q_{2}\right) \leq \max \left(d_{\Pi}\left(\left\langle\left\langle q_{1}\right\rangle\right\rangle_{1},\left\langle\left\langle q_{2}\right\rangle\right\rangle_{2}\right),\right. \\
&\left.\sup _{q_{1} \stackrel{\sigma}{\rightarrow} q_{1}^{\prime} q_{2}{ }_{q_{2} \rightarrow}^{\rightarrow} q_{2}^{\prime}} f_{\mathcal{S}}^{\min }\left(q_{1}^{\prime}, q_{2}^{\prime}\right)\right)
\end{aligned}
$$

When $i$ tends to $+\infty$, this inequality becomes

$$
\begin{aligned}
& f_{\mathcal{S}}^{\min }\left(q_{1}, q_{2}\right) \leq \max \left(d_{\Pi}\left(\left\langle\left\langle q_{1}\right\rangle\right\rangle_{1},\left\langle\left\langle q_{2}\right\rangle\right\rangle_{2}\right),\right. \\
&\left.\sup _{q_{1} \stackrel{\sigma}{\rightarrow}_{1} q_{1}^{\prime} q_{2} \stackrel{\sigma}{\rightarrow}_{2} q_{2}^{\prime}} f_{\mathcal{S}}^{\min }\left(q_{1}^{\prime}, q_{2}^{\prime}\right)\right)
\end{aligned}
$$

Since for all $\left(q_{1}, q_{2}\right) \in Q_{1} \times Q_{2}$, the sequence $\left\{f_{\mathcal{S}}^{i}\left(q_{1}, q_{2}\right)\right\}_{i \in \mathbb{N}}$ is increasing, then the sequence

$$
\left\{\sup _{q_{1} \stackrel{\sigma}{\rightarrow}_{1} q_{1}^{\prime}} \inf _{q_{2} \stackrel{\sigma}{\rightarrow}_{2} q_{2}^{\prime}} f_{\mathcal{S}}^{i}\left(q_{1}^{\prime}, q_{2}^{\prime}\right)\right\}_{i \in \mathbb{N}}
$$

is increasing as well. Let $l\left(q_{1}, q_{2}\right) \in \mathbb{R}^{+} \cup\{+\infty\}$ denote the limit of this sequence. For all $i \in \mathbb{N}$

$$
\sup _{\substack{\sigma \\ q_{1} \stackrel{\sigma}{\rightarrow}_{1} q_{1}^{\prime}}} \inf _{q_{2} \stackrel{\sigma}{\rightarrow}_{2} q_{2}^{\prime}} f_{\mathcal{S}}^{i}\left(q_{1}^{\prime}, q_{2}^{\prime}\right) \leq l\left(q_{1}, q_{2}\right) .
$$

Let $q_{1} \stackrel{\sigma}{\rightarrow}_{1} q_{1}^{\prime}$, for all $i \in \mathbb{N}$, for all $\varepsilon>0$, there exists $q_{2} \stackrel{\sigma}{\rightarrow}{ }_{2} q_{2}^{\prime i}$, such that $f_{\mathcal{S}}^{i}\left(q_{1}^{\prime}, q_{2}^{\prime}\right) \leq l\left(q_{1}, q_{2}\right)+\varepsilon$. From Lemma 11, it follows that for all $i \in \mathbb{N}$, there exists $q_{2} \stackrel{\sigma}{\rightarrow}_{2} q_{2}^{\prime i}$, such that $f_{\mathcal{S}}^{i}\left(q_{1}^{\prime}, q_{2}^{\prime i}\right) \leq$ $l\left(q_{1}, q_{2}\right) . \operatorname{Post}_{2}^{\sigma}\left(q_{2}\right)$ is compact, then there exists $\left\{q_{2}^{\prime i_{k}}\right\}_{k \in \mathbb{N}}$ a subsequence of $\left\{q_{2}^{\prime i}\right\}_{i \in \mathbb{N}}$ which converges to $q_{2}^{\prime} \in \operatorname{Post}_{2}^{\sigma}\left(q_{2}\right)$. Let $i \in \mathbb{N}$, there exists $n \in \mathbb{N}$, such that for all $k \geq n, i_{k} \geq i$. Hence for all $k \geq n, f_{\mathcal{S}}^{i}\left(q_{1}^{\prime}, q_{2}^{\prime i_{k}}\right) \leq f_{\mathcal{S}}^{i_{k}}\left(q_{1}^{\prime}, q_{2}^{\prime i_{k}}\right) \leq l\left(q_{1}, q_{2}\right)$. Since this holds for all $k \geq n$, we have $f_{\mathcal{S}}^{i}\left(q_{1}^{\prime}, q_{2}^{\prime}\right) \leq l\left(q_{1}, q_{2}\right)$. This holds for all $i \in \mathbb{N}$ and hence, $f_{\mathcal{S}}^{\min }\left(q_{1}^{\prime}, q_{2}^{\prime}\right) \leq l\left(q_{1}, q_{2}\right)$.
We proved that for all $q_{1} \stackrel{\sigma}{\rightarrow} 1 q_{1}^{\prime}$, there exists $q_{2} \stackrel{\sigma}{\rightarrow} 2 q_{2}^{\prime}$, such that $f_{\mathcal{S}}^{\min }\left(q_{1}^{\prime}, q_{2}^{\prime}\right) \leq l\left(q_{1}, q_{2}\right)$. Therefore

$$
\sup _{\substack{\sigma \\ q_{1} \rightarrow} q_{1}^{\prime}} \inf _{q_{2} \stackrel{\sigma}{\rightarrow}{ }_{2} q_{2}^{\prime}} f_{\mathcal{S}}^{\min }\left(q_{1}^{\prime}, q_{2}^{\prime}\right) \leq l\left(q_{1}, q_{2}\right) .
$$

Hence

$$
\begin{aligned}
& f_{\mathcal{S}}^{\min }\left(q_{1}, q_{2}\right) \geq \max \left(d_{\Pi}\left(\left\langle\left\langle q_{1}\right\rangle\right\rangle_{1},\left\langle\left\langle q_{2}\right\rangle\right\rangle_{2}\right)\right. \\
&\left.\sup _{q_{1} \stackrel{\sigma}{\rightarrow}_{1} q_{1}^{\prime}} \inf _{q_{2} \stackrel{\sigma}{\rightarrow}_{2} q_{2}^{\prime}} f_{\mathcal{S}}^{\min }\left(q_{1}^{\prime}, q_{2}^{\prime}\right)\right) .
\end{aligned}
$$

Now, let us prove that $f_{\mathcal{S}}{ }^{\text {min }}$ is the smallest function satisfying (5). Let $f$ be a solution of (5), then for all $\left(q_{1}, q_{2}\right) \in Q_{1} \times Q_{2}$, $f\left(q_{1}, q_{2}\right) \geq d_{\Pi}\left(\left\langle\left\langle q_{1}\right\rangle\right\rangle_{1},\left\langle\left\langle q_{2}\right\rangle\right\rangle_{2}\right)=f_{\mathcal{S}}^{0}\left(q_{1}, q_{2}\right)$. By induction, it is easy to show that for all $i \in \mathbb{N} f\left(q_{1}, q_{2}\right) \geq f_{\mathcal{S}}^{i}\left(q_{1}, q_{2}\right)$ and hence $f\left(q_{1}, q_{2}\right) \geq f_{\mathcal{S}}^{\min }\left(q_{1}, q_{2}\right)$.

Let us remark that the directed branching distance $f_{\mathcal{S}}^{\min }$ is the smallest solution of the fixed-point (5) in the sense that for all solution $f$ of (5), for all $\left(q_{1}, q_{2}\right) \in Q_{1} \times Q_{2}, f_{\mathcal{S}}^{\min }\left(q_{1}, q_{2}\right) \leq$ $f\left(q_{1}, q_{2}\right)$.

We now arrive to the main result of this section which states that for regular metric transition systems, the simulation metric can be computed by solving a static game where the cost function of the game is the directed branching distance.

Theorem 16: Let $f_{\mathcal{S}}^{\min }$ be the directed branching distance between $T_{1}$ and $T_{2}$. Then

$$
d_{\mathcal{S}}\left(T_{1}, T_{2}\right)=\sup _{q_{1} \in Q_{1}^{0}} \inf _{q_{2} \in Q_{2}^{0}} f_{\mathcal{S}}^{\min }\left(q_{1}, q_{2}\right) .
$$

Proof: Let $\delta>d_{\mathcal{S}}\left(T_{1}, T_{2}\right)$, then $T_{1} \preceq_{\delta} T_{2}$. Hence, for all $q_{1} \in Q_{1}^{0}$, there exists $q_{2} \in Q_{2}^{0}$, such that $\left(q_{1}, q_{2}\right) \in \mathcal{S}_{\delta}^{\max }$. From Theorem 14 , it follows that $f_{\mathcal{S}}^{\min }\left(q_{1}, q_{2}\right) \leq \delta$. Consequently

$$
\sup _{q_{1} \in Q_{1}^{0}} \inf _{q_{2} \in Q_{2}^{0}} f_{\mathcal{S}}^{\min }\left(q_{1}, q_{2}\right) \leq \delta
$$

Since this holds for all $\delta>d_{\mathcal{S}}\left(T_{1}, T_{2}\right)$

$$
\sup _{q_{1} \in Q_{1}^{0}} \inf _{q_{2} \in Q_{2}^{0}} f_{\mathcal{S}}^{\min }\left(q_{1}, q_{2}\right) \leq d_{\mathcal{S}}\left(T_{1}, T_{2}\right)
$$

Conversely, let

$$
\delta=\sup _{q_{1} \in Q_{1}^{0}} \inf _{q_{2} \in Q_{2}^{0}} f_{\mathcal{S}}^{\min }\left(q_{1}, q_{2}\right)
$$

Let $q_{1} \in Q_{1}^{0}$, then for all $\varepsilon>0$, there exists $q_{2} \in Q_{2}^{0}$ such that, $f_{\mathcal{S}}^{\min }\left(q_{1}, q_{2}\right) \leq \delta+\varepsilon$. From Lemma 11, there exists $q_{2} \in Q_{2}^{0}$ such that, $f_{\mathcal{S}}^{\min }\left(q_{1}, q_{2}\right) \leq \delta$. Hence, for all $q_{1} \in Q_{1}^{0}$, there exists $q_{2} \in Q_{2}^{0}$, such that $\left(q_{1}, q_{2}\right) \in \mathcal{S}_{\delta}^{\max }$. Consequently, $T_{1} \preceq_{\delta} T_{2}$ and, therefore, $d_{\mathcal{S}}\left(T_{1}, T_{2}\right) \leq \delta$. 
To summarize, in order to exactly compute the simulation metric, one must solve (5) in order to obtain the branching distance, and then solve the much easier static game (6). In Section VII, we will consider relaxations of (5), but we first develop analogous results for exactly computing the bisimulation metric.

\section{Maximal Approximate Bisimulations}

The development of this section is similar to the exact computation of the simulation metric and therefore the proofs in this section are omitted. The well known bisimulation algorithm [17], can be generalized for approximate bisimulations as follows.

Algorithm 3: Let $T_{1}, T_{2} \in \mathcal{T}_{M}^{*}(\Sigma, \Pi)$. For $\delta \geq 0$, define the following sequence $\left\{\mathcal{B}_{\delta}^{i}\right\}_{i \in \mathbb{N}}$ of subsets of $\mathcal{Q}_{1} \times \mathcal{Q}_{2}$ :

$$
\begin{aligned}
\mathcal{B}_{\delta}^{0} & =\left\{\left(q_{1}, q_{2}\right) \in \mathcal{Q}_{1} \times \mathcal{Q}_{2} \mid d_{\Pi}\left(\left\langle\left\langle q_{1}\right\rangle\right\rangle_{1},\left\langle\left\langle q_{2}\right\rangle\right\rangle_{2}\right) \leq \delta\right\} \\
\mathcal{B}_{\delta}^{i+1} & =\left\{\begin{array}{l|l}
\forall q_{1} \stackrel{\sigma}{\rightarrow} q_{1}^{\prime}, \exists q_{2} \stackrel{\sigma}{\rightarrow}_{2} q_{2}^{\prime}, \\
\left(q_{1}, q_{2}\right) \in \mathcal{B}_{\delta}^{i} & \begin{array}{l}
\text { such that }\left(q_{1}^{\prime}, q_{2}^{\prime}\right) \in \mathcal{B}_{\delta}^{i} \\
\forall q_{2} \underset{\rightarrow}{\rightarrow} q_{2}^{\prime}, \exists q_{1} \underset{\rightarrow}{\rightarrow} q_{1}^{\prime}, \\
\text { such that }\left(q_{1}^{\prime}, q_{2}^{\prime}\right) \in \mathcal{B}_{\delta}^{i}
\end{array}
\end{array}\right\} .
\end{aligned}
$$

The previous algorithm approaches the maximal (coarsest) approximate bisimulation relation $\mathcal{B}_{\delta}^{\max }$.

Theorem 17: Let $\left\{\mathcal{B}_{\delta}^{i}\right\}_{i \in \mathbb{N}}$ be the decreasing sequence of sets defined by Algorithm 3 and $\mathcal{B}_{\delta}^{\max }$ be the maximal $\delta$-approximate bisimulation relation between $T_{1}$ and $T_{2}$. Then, the following properties hold:

$$
\forall i \in \mathbb{N}, \mathcal{B}_{\delta}^{\max } \subseteq \mathcal{B}_{\delta}^{i} \bigcap_{i=0}^{i=+\infty} \mathcal{B}_{\delta}^{i}=\mathcal{B}_{\delta}^{\max }
$$

\section{Branching Distance}

If we represent approximate simulation relations as levels sets of functions, then the following dual approach based on functions can be used for fixed-point computation.

Algorithm 4: Let $T 1, T_{2} \in \mathcal{T}_{M}^{*}(\Sigma, \Pi)$. Define the following sequence $\left\{f_{\mathcal{B}}^{i}\right\}_{i \in \mathbb{N}}$ of functions from $\mathcal{Q}_{1} \times \mathcal{Q}_{2}$ to $\mathbb{R}^{+} \cup\{+\infty\}$

$$
\begin{aligned}
& f_{\mathcal{B}}^{0}\left(q_{1}, q_{2}\right)=d_{\Pi}\left(\left\langle\left\langle q_{1}\right\rangle\right\rangle_{1},\left\langle\left\langle q_{2}\right\rangle\right\rangle_{2}\right) \\
& f_{\mathcal{B}}^{i+1}\left(q_{1}, q_{2}\right)=\max \left(d_{\Pi}\left(\left\langle\left\langle q_{1}\right\rangle\right\rangle_{1},\left\langle\left\langle q_{2}\right\rangle\right\rangle_{2}\right)\right. \text {, } \\
& \sup _{\substack{\sigma \\
q_{1} \rightarrow{ }_{1} q_{1}^{\prime}}} \inf _{q_{2} \rightarrow_{2} q_{2}^{\prime}} f_{\mathcal{B}}^{i}\left(q_{1}^{\prime}, q_{2}^{\prime}\right), \\
& \left.\sup _{\substack{\sigma \\
q_{2} \rightarrow 2 q_{2}^{\prime}}} \inf _{q_{1}{ }^{\sigma}{ }_{1} q_{1}^{\prime}} f_{\mathcal{B}}^{i}\left(q_{1}^{\prime}, q_{2}^{\prime}\right)\right), \quad i \in \mathbb{N} \text {. }
\end{aligned}
$$

As for the case of approximate simulation, we can show that for all $\left(q_{1}, q_{2}\right) \in Q_{1} \times Q_{2}$, the series $\left\{f_{\mathcal{B}}^{i}\left(q_{1}, q_{2}\right)\right\}_{i \in \mathbb{N}}$ is increasing. Hence, the sequence of functions $\left\{f_{\mathcal{B}}^{i}\right\}_{i \in \mathbb{N}}$ converges pointwise in $\mathbb{R}^{+} \cup\{+\infty\}$.
Definition 19: Let $\left\{f_{\mathcal{B}}^{i}\right\}_{i \in \mathbb{N}}$ be the sequence of functions defined by Algorithm 4. The branching distance [16] between $T_{1}$ and $T_{2}$ is the function defined by

$$
\forall\left(q_{1}, q_{2}\right) \in Q_{1} \times Q_{2}, f_{\mathcal{B}}^{\min }\left(q_{1}, q_{2}\right)=\lim _{i \rightarrow \infty} f_{\mathcal{B}}^{i}\left(q_{1}, q_{2}\right) .
$$

The duality between the approach using relations and the approach using functions is captured by the following result.

Theorem 18: Let $\left\{\mathcal{B}_{\delta}^{i}\right\}_{i \in \mathbb{N}}$ be the sequence of sets defined by Algorithm 3 and $\left\{f_{\mathcal{B}}^{i}\right\}_{i \in \mathbb{N}}$ be the sequence of functions defined by Algorithm 4. Then, for all $i \in N$

$$
\forall \delta \geq 0, \mathcal{B}_{\delta}^{i}=\left\{\left(q_{1}, q_{2}\right) \in Q_{1} \times Q_{2} \mid f_{\mathcal{B}}^{i}\left(q_{1}, q_{2}\right) \leq \delta\right\} .
$$

Let $\mathcal{B}_{\delta}^{\max }$ be the maximal $\delta$-approximate bisimulation relation between $T_{1}$ and $T_{2}$ and $f_{\mathcal{B}}^{\min }$ be the branching distance between $T_{1}$ and $T_{2}$. Then

$$
\forall \delta \geq 0, \mathcal{B}_{\delta}^{\max }=\left\{\left(q_{1}, q_{2}\right) \in Q_{1} \times Q_{2} \mid f_{\mathcal{B}}^{\min }\left(q_{1}, q_{2}\right) \leq \delta\right\} .
$$

The branching distance is the smallest solution of the fixedpoint equation given by the following theorem.

Theorem 19: The branching distance between $T_{1}$ and $T_{2}$ is the smallest function defined on $Q_{1} \times Q_{2}$ with values in $\mathbb{R}^{+} \cup$ $\{+\infty\}$ satisfying the following functional equation:

$$
\begin{aligned}
& f_{\mathcal{B}}^{\min }\left(q_{1}, q_{2}\right)=\max \left(d_{\Pi}\left(\left\langle\left\langle q_{1}\right\rangle\right\rangle_{1},\left\langle\left\langle q_{2}\right\rangle\right\rangle_{2}\right),\right.
\end{aligned}
$$

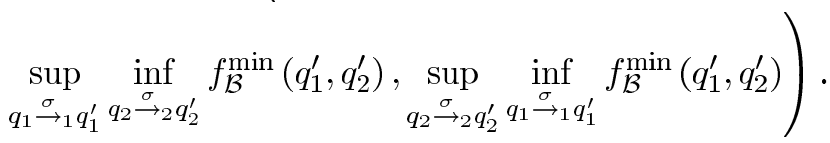

Finally, similar to the simulation metric, for regular metric transition systems, the bisimulation metric can be computed by solving a static game where the cost function of the game is the branching distance.

Theorem 20: Let $f_{\mathcal{B}}^{\min }$ be the branching distance between $T_{1}$ and $T_{2}$. Then,

$$
\begin{aligned}
d_{\mathcal{B}}\left(T_{1}, T_{2}\right)=\max \left(\sup _{q_{1} \in Q_{1}^{0}} \inf _{q_{2} \in Q_{2}^{0}} f_{\mathcal{B}}^{\min }\left(q_{1}, q_{2}\right),\right. \\
\left.\qquad \sup _{q_{2} \in Q_{2}^{0}} \inf _{q_{1} \in Q_{1}^{0}} f_{\mathcal{B}}^{\min }\left(q_{1}, q_{2}\right)\right) .
\end{aligned}
$$

In this section, we proposed a method for the exact computation of the simulation and the bisimulation metrics between regular metric transition systems. It consists in solving a static game where the cost function is the branching distance (see Theorems 16 and 20). For systems with a finite number of states, fixed point Algorithms 2 and 4 for the computation of the branching distance are guaranteed to terminate within a finite number of steps. For systems with an infinite number of states, these algorithms do not necessarily reach a fixed point in a finite number of iterations. Then, an alternative approach is to solve directly the functional equations given by Theorems 15 and 19. However, in cases where the equations given by Theorems 15 and 
19 are difficult to solve, one can consider the relaxation that are proposed in the following section.

\section{APPROXIMATE METRIC COMPUTATION}

One of the great advantages of having metric structure on transition systems is that metrics enable us to consider relaxations. If the equations given by Theorems 15 and 19 are difficult to solve, then we can consider relaxations that will result in computing an over-approximation of the simulation or the bisimulation metrics. The relaxations we propose are based on classes of functions that we call simulation and bisimulation functions.

\section{A. Simulation Functions}

Let $T_{1}=\left(Q_{1}, \Sigma, \rightarrow_{1}, Q_{1}^{0}, \Pi,\langle\langle\cdot\rangle\rangle_{1}\right)$ and $T_{2}=\left(Q_{2}, \Sigma, \rightarrow_{2}\right.$ $\left., Q_{2}^{0}, \Pi,\langle\langle\cdot\rangle\rangle_{2}\right)$ be two elements of $\mathcal{T}_{M}^{*}(\Sigma, \Pi) .^{5}$ A simulation function between $T_{1}$ and $T_{2}$ is a positive function defined on $Q_{1} \times Q_{2}$, bounding the distance between the observations associated to the couple $\left(q_{1}, q_{2}\right)$ and non increasing under the dynamics of the systems.

Definition 20 (Simulation Functions): A function $f_{\mathcal{S}}: Q_{1} \times$ $Q_{2} \rightarrow \mathbb{R}^{+} \cup\{+\infty\}$ is called a simulation function between $T_{1}$ and $T_{2}$ if its level sets are closed, and for all $\left(q_{1}, q_{2}\right) \in Q_{1} \times Q_{2}$ :

$$
\begin{aligned}
& f_{\mathcal{S}}\left(q_{1}, q_{2}\right) \geq \max \left(d_{\Pi}\left(\left\langle\left\langle q_{1}\right\rangle\right\rangle_{1},\left\langle\left\langle q_{2}\right\rangle\right\rangle_{2}\right),\right. \\
& \left.\sup _{q_{1} \stackrel{\sigma}{\rightarrow}_{1} q_{1}^{\prime}} \inf _{q_{2} \stackrel{\sigma}{\rightarrow}_{2} q_{2}^{\prime}} f_{\mathcal{S}}\left(q_{1}^{\prime}, q_{2}^{\prime}\right)\right) \text {. }
\end{aligned}
$$

For regular metric labeled transition systems, simulation functions are reminiscent of (robust) Lyapunov functions and can be seen as relaxations of the directed branching distance. In fact, the directed branching distance is a simulation function itself.

Theorem 21: Let $T_{1}, T_{2} \in \mathcal{T}_{M}^{*}(\Sigma, \Pi)$ and let $f_{\mathcal{S}}^{\min }$ be the directed branching distance between $T_{1}$ and $T_{2}$. Then, $f_{\mathcal{S}}^{\min }$ is the smallest simulation function between $T_{1}$ and $T_{2}$.

Proof: We know that $f_{S}^{\min }$ has closed level sets. From Theorem 15 , it is clear that $f_{\mathcal{S}}^{\min }$ is a simulation function. Let $f_{\mathcal{S}}$ be a simulation function between $T_{1}$ and $T_{2}$, let $\left\{f_{\mathcal{S}}^{i}\right\}_{i \in \mathbb{N}}$ be the sequence of functions defined by Algorithm 2. We have, for all $\left(q_{1}, q_{2}\right) \in Q_{1} \times Q_{2}, f_{\mathcal{S}}\left(q_{1}, q_{2}\right) \geq d_{\Pi}\left(\left\langle\left\langle q_{1}\right\rangle\right\rangle_{1},\left\langle\left\langle q_{2}\right\rangle\right\rangle_{2}\right)=$ $f_{\mathcal{S}}^{0}\left(q_{1}, q_{2}\right)$. By induction, it is easy to show that for all $\left(q_{1}, q_{2}\right) \in$ $Q_{1} \times Q_{2}$, for all $i \in \mathbb{N}, f_{\mathcal{S}}\left(q_{1}, q_{2}\right) \geq f_{\mathcal{S}}^{i}\left(q_{1}, q_{2}\right)$ and hence $f_{\mathcal{S}}\left(q_{1}, q_{2}\right) \geq f_{\mathcal{S}}^{\min }\left(q_{1}, q_{2}\right)$.

As in Theorem 15, the directed branching distance $f_{\mathcal{S}}^{\min }$ is the smallest simulation function between $T_{1}$ and $T_{2}$ in the sense that for all simulation function $f_{\mathcal{S}}$, for all $\left(q_{1}, q_{2}\right) \in Q_{1} \times Q_{2}$, $f_{\mathcal{S}}^{\min }\left(q_{1}, q_{2}\right) \leq f_{\mathcal{S}}\left(q_{1}, q_{2}\right)$. Thus, the directed branching distance between $T_{1}$ and $T_{2}$ will be also called minimal simulation function between $T_{1}$ and $T_{2}$.

A simulation function between $T_{1}$ and $T_{2}$ is a convenient way to define a family $\left\{\mathcal{S}_{\delta}\right\}_{\delta>0}$ of approximate simulation relations of $T_{1}$ by $T_{2}$.

\footnotetext{
${ }^{5}$ Even though we do not need to assume that $T_{1}$ and $T_{2}$ are regular, we do have to assume that the successor maps have compact images.
}

Theorem 22: Let $f_{\mathcal{S}}$ be a simulation function between $T_{1}$ and $T_{2}$. Then, for all $\delta \geq 0$

$$
\mathcal{S}_{\delta}=\left\{\left(q_{1}, q_{2}\right) \in Q_{1} \times Q_{2} \mid f_{\mathcal{S}}\left(q_{1}, q_{2}\right) \leq \delta\right\}
$$

is a $\delta$-approximate simulation relation of $T_{1}$ by $T_{2}$.

Proof: Let $\left(q_{1}, q_{2}\right) \in \mathcal{S}_{\delta}$, then $d_{\Pi}\left(\left\langle\left\langle q_{1}\right\rangle\right\rangle_{1},\left\langle\left\langle q_{2}\right\rangle\right\rangle_{2}\right) \leq$ $f_{\mathcal{S}}\left(q_{1}, q_{2}\right) \leq \delta$. Let $q_{1} \stackrel{\sigma}{\rightarrow}_{1} q_{1}^{\prime}$, then for all $\varepsilon>0$, there exists $q_{2} \stackrel{\sigma}{\rightarrow}_{2} q_{2}^{\prime}$ such that $f_{\mathcal{S}}\left(q_{1}^{\prime}, q_{2}^{\prime}\right) \leq f_{\mathcal{S}}\left(q_{1}, q_{2}\right)+\varepsilon \leq \delta+\varepsilon$. From Lemma 11 , there exists $q_{2} \stackrel{\sigma_{2}}{\rightarrow} q_{2}^{\prime}$ such that $f_{\mathcal{S}}\left(q_{1}^{\prime}, q_{2}^{\prime}\right) \leq \delta$. Hence $\mathcal{S}_{\delta}$ is a $\delta$-approximate simulation relation of $T_{1}$ by $T_{2}$.

Let us remark that particularly the zero set of a simulation function is an exact simulation relation. We can now state the following result which shows that an over-approximation of the simulation metric can be computed by solving a game where the cost function is a simulation function.

Theorem 23: Let $f_{\mathcal{S}}$ be any simulation function between $T_{1}$ and $T_{2}$. Then

$$
d_{\mathcal{S}}\left(T_{1}, T_{2}\right) \leq \sup _{q_{1} \in Q_{1}^{0}} \inf _{q_{2} \in Q_{2}^{0}} f_{\mathcal{S}}\left(q_{1}, q_{2}\right)
$$

Proof: Let

$$
\delta=\sup _{q_{1} \in Q_{1}^{0}} \inf _{q_{2} \in Q_{2}^{0}} f_{\mathcal{S}}^{\min }\left(q_{1}, q_{2}\right)
$$

Let $q_{1} \in Q_{1}^{0}$, then for all $\varepsilon>0$, there exists $q_{2} \in Q_{2}^{0}$ such that, $f_{\mathcal{S}}^{\min }\left(q_{1}, q_{2}\right) \leq \delta+\varepsilon$. Hence, for all $\varepsilon>0, T_{1} \preceq_{\delta+\varepsilon} T_{2}$. Therefore, $d_{\mathcal{S}}\left(T_{1}, T_{2}\right) \leq \delta$.

The above theorem enables us to over-approximate the simulation metric by relaxing the solution of (5) with Lyapunov-like simulation functions. In addition to this relaxation, the following result shows that, for the synchronous composition defined in Section V, simulation functions are also compositional. The proof can be found in [34].

Theorem 24: Let $f_{\mathcal{S}_{1}}$ be a simulation function of $T_{1}$ by $U_{1}$ and $f_{\mathcal{S}_{2}}$ be a simulation function of $T_{2}$ by $U_{2}$, then $f_{\mathcal{S}}=f_{\mathcal{S}_{1}}+$ $f_{\mathcal{S}_{2}}$ is a simulation function of $T_{1} \| T_{2}$ by $U_{1} \| U_{2}$.

\section{B. Bisimulation Functions}

We now consider similar relaxations for the bisimulation metric. Bisimulation functions are defined in a similar way to simulation functions. The proofs of the results of this part are omitted because they are similar to the proofs for simulation functions.

Definition 21 (Bisimulation Functions): A function $f_{\mathcal{B}}: Q_{1} \times Q_{2} \rightarrow \mathbb{R}^{+} \cup\{+\infty\}$ is a bisimulation function between $T_{1}$ and $T_{2}$ if its level sets are closed and for all $\left(q_{1}, q_{2}\right) \in Q_{1} \times Q_{2}$ :

$f_{\mathcal{B}}\left(q_{1}, q_{2}\right) \geq \max \left(d_{\Pi}\left(\left\langle\left\langle q_{1}\right\rangle\right\rangle_{1},\left\langle\left\langle q_{2}\right\rangle\right\rangle_{2}\right)\right.$,

$$
\left.\sup _{q_{1} \stackrel{\sigma}{\rightarrow}{ }_{1} q_{1}^{\prime}} \inf _{q_{2} \stackrel{\sigma}{\rightarrow} 2 q_{2}^{\prime}} f_{\mathcal{B}}\left(q_{1}^{\prime}, q_{2}^{\prime}\right), \sup _{q_{2} \stackrel{\sigma}{\rightarrow}_{2} q_{2}^{\prime}} \inf _{q_{1} \stackrel{\sigma}{\rightarrow}_{1} q_{1}^{\prime}} f_{\mathcal{B}}\left(q_{1}^{\prime}, q_{2}^{\prime}\right)\right) \text {. }
$$


For regular metric labeled transition systems, we can show that the branching distance is a bisimulation function.

Theorem 25: Let $T_{1}, T_{2} \in \mathcal{T}_{M}^{*}(\Sigma, \Pi)$, let $f_{\mathcal{B}}^{\min }$ be the branching distance between $T_{1}$ and $T_{2}$. Then, $f_{\mathcal{B}}^{\min }$ is the smallest bisimulation function between $T_{1}$ and $T_{2}$.

Thus, the directed branching distance between $T_{1}$ and $T_{2}$ will be also called minimal bisimulation function between $T_{1}$ and $T_{2}$.

Theorem 26: Let $f_{\mathcal{B}}$ be a bisimulation function between $T_{1}$ and $T_{2}$, then for all $\delta \geq 0$,

$$
\mathcal{B}_{\delta}=\left\{\left(q_{1}, q_{2}\right) \in Q_{1} \times Q_{2} \mid f_{\mathcal{B}}\left(q_{1}, q_{2}\right) \leq \delta\right\}
$$

is a $\delta$-approximate bisimulation relation of $T_{1}$ by $T_{2}$.

Particularly the zero set of a bisimulation function is an exact bisimulation relation.

Theorem 27: Let $f_{\mathcal{B}}$ be a bisimulation function between $T_{1}$ and $T_{2}$. Then,

$$
\begin{aligned}
d_{\mathcal{B}}\left(T_{1}, T_{2}\right) \leq \max \left(\sup _{q_{1} \in Q_{1}^{0}} \inf _{q_{2} \in Q_{2}^{0}} f_{\mathcal{B}}\left(q_{1}, q_{2}\right)\right. \\
\left.\qquad \sup _{q_{2} \in Q_{2}^{0}} \inf _{q_{1} \in Q_{1}^{0}} f_{\mathcal{B}}\left(q_{1}, q_{2}\right)\right) .
\end{aligned}
$$

The following theorem states that bisimulation functions are compositional.

Theorem 28: Let $f_{\mathcal{B}_{1}}$ be a bisimulation function between $T_{1}$ and $U_{1}$ and $f_{\mathcal{B}_{2}}$ be a bisimulation function between $T_{2}$ and $U_{2}$, then $f_{\mathcal{B}}=f_{\mathcal{B}_{1}}+f_{\mathcal{B}_{2}}$ is a bisimulation function between $T_{1} \| T_{2}$ and $U_{1} \| U_{2}$.

In this section, we proposed Lyapunov-like relaxations for computing over-approximations of the simulation and the bisimulation metrics, which can further overapproximate the language and reachability metric between two transition systems. In the final section, we illustrate how these computations could be used for reducing the complexity of safety verification problems for continuous systems.

\section{VERIFICATION ILLUSTRATION}

Despite significant progress in the formal verification of discrete systems [3], the progress for continuous (and thus hybrid) systems has been limited to systems of small continuous dimension. The Lyapunov-like relaxations of Section VII allow us to use a wealth of Lyapunov techniques for approximating simulation and bisimulation functions. We present two examples, one simply illustrating the steps of our framework for nondeterministic linear systems, and one showing how Lyapunov equations can dramatically reduce the complexity of safety verification problems for deterministic linear systems with an approximation error that is easily computable and acceptable.

\section{A. Nondeterministic Continuous Systems}

Consider the following continuous-time linear system with bounded disturbances:

$$
\left\{\begin{array}{l}
\dot{x}_{1}(t)=-2 x_{1}(t)+y_{1}(t)+z_{1}(t)+d_{1}(t) \\
\dot{y}_{1}(t)=-x_{1}(t)+z_{1}(t)+d_{1}(t) \\
\dot{z}_{1}(t)=x_{1}(t)-y_{1}(t)-2 z_{1}(t)
\end{array}\right.
$$

The system is observed through the variable $\pi_{1}(t)=x_{1}(t)$. The values of the disturbance $d_{1}($.$) is constrained in the set [-1,1]$. The initial state lies in the polytope $I_{1}$ given by

$$
I_{1}=\left\{\begin{array}{l|l}
\left(x_{1}, y_{1}, z_{1}\right) \in \mathbb{R}^{3} & \begin{array}{c}
-1 \leq x_{1}-y_{1}-z_{1} \leq 1 \\
8 \leq y_{1} \leq 9,-6 \leq z_{1} \leq-4
\end{array}
\end{array}\right\} .
$$

As stated previously, we can derive a regular metric transition system $T_{1} \in \mathcal{T}_{M}^{*}\left(\mathbb{R}^{+}, \mathbb{R}\right)$ which is also nondeterministic. We want to show that $T_{1}$ can be approximated by the regular metric labeled transition system $T_{2} \in \mathcal{T}_{M}^{*}\left(\mathbb{R}^{+}, \mathbb{R}\right)$ generated by the following linear system:

$$
\dot{x}_{2}(t)=-x_{2}(t)+d_{2}(t) .
$$

The system is observed through the variable $\pi_{2}(t)=x_{2}(t)$. The values of the disturbance $d_{2}($.$) are constrained in the set$ $[-1,1]$. The initial value of the state variable lies in the interval $I_{2}=[2,5]$. Let us show that

$$
f_{\mathcal{B}}\left(x_{1}, y_{1}, z_{1}, x_{2}\right)=\left|x_{1}-y_{1}-z_{1}\right|+\left|y_{1}+z_{1}-x_{2}\right|
$$

is a bisimulation function between $T_{1}$ and $T_{2}$. First, let us remark that from the triangular inequality, it follows that

$$
\left|x_{1}-x_{2}\right| \leq f_{\mathcal{B}}\left(x_{1}, y_{1}, z_{1}, x_{2}\right) \text {. }
$$

Hence, $f_{\mathcal{B}}\left(x_{1}, y_{1}, z_{1}, x_{2}\right)$ bounds the distance between the observations of $T_{1}$ and $T_{2}$. Moreover, we can check that

$$
\begin{array}{r}
\frac{\partial f_{\mathcal{B}}}{\partial x_{1}} \dot{x}_{1}+\frac{\partial f_{\mathcal{B}}}{\partial y_{1}} \dot{y}_{1}+\frac{\partial f_{\mathcal{B}}}{\partial z_{1}} \dot{z}_{1}+\frac{\partial f_{\mathcal{B}}}{\partial x_{2}} \dot{x}_{2}=-2\left|x_{1}-y_{1}-z_{1}\right| \\
-\left|y_{1}+z_{1}-x_{2}\right|+\left(d_{1}-d_{2}\right) \operatorname{sgn}\left(y_{1}+z_{1}-x_{2}\right) .
\end{array}
$$

Hence, for all disturbance $d_{1}(t)$ (respectively $d_{2}(t)$ ) there exists a disturbance $d_{2}(t)$ (respectively $d_{1}(t)$ ) such that $d f_{\mathcal{B}}\left(x_{1}(t), y_{1}(t), z_{1}(t), x_{2}(t)\right) / d t$ is negative. Therefore, $f_{\mathcal{B}}$ is non increasing under the dynamics of the systems which implies that $f_{\mathcal{B}}$ is a bisimulation function between $T_{1}$ and $T_{2}$.

From Theorem 27, an over-approximation of the bisimulation metric between $T_{1}$ and $T_{2}$ can be computed by solving a game. We can check that

$$
\sup _{\left(x_{1}, y_{1}, z_{1}\right) \in I_{1}} \inf _{x_{2} \in I_{2}} f_{\mathcal{B}}\left(x_{1}, y_{1}, z_{1}, x_{2}\right)=1
$$

and that

$$
\sup _{x_{2} \in I_{2}} \inf _{\left(x_{1}, y_{1}, z_{1}\right) \in I_{1}} f_{\mathcal{B}}\left(x_{1}, y_{1}, z_{1}, x_{2}\right)=0 .
$$



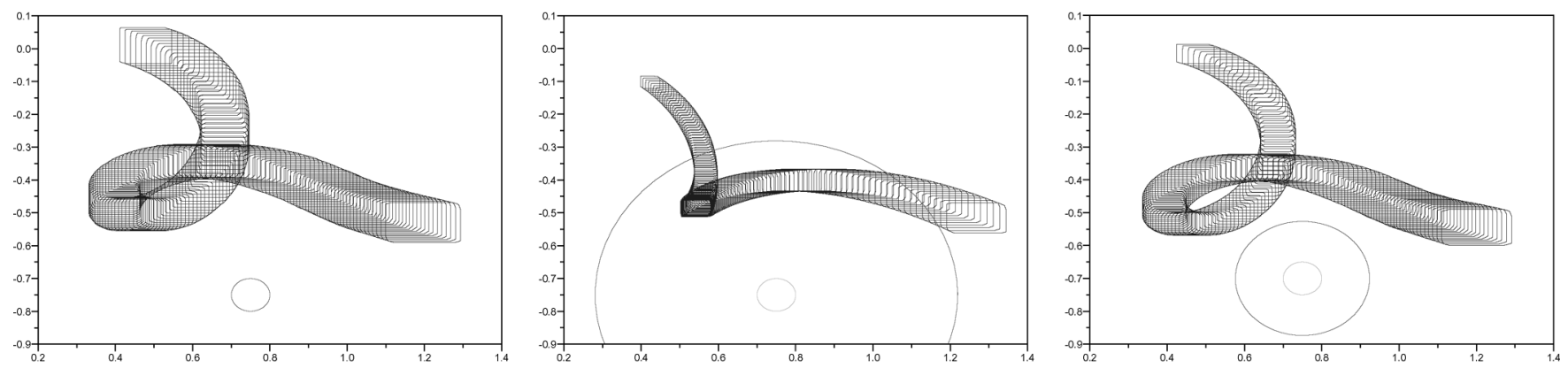

Fig. 1. Reachable sets of the original hundred dimensional system (left) and of its six-dimensional and ten-dimensional approximations (center and right). The circle on the left figure and the inner circle on the others represent the unsafe set $\Pi_{U}$. The outer circle on the center and right figure consists of the set of points whose distance to $\Pi_{U}$ is smaller than the upper bound of the bisimulation metric.

Hence, $d_{\mathcal{B}}\left(T_{1}, T_{2}\right) \leq 1$. The systems $T_{1}$ and $T_{2}$ are approximately bisimilar with the precision 1 . We now propose to use this result to compute an over-approximation of the reachable set of $T_{1}$. From Theorem 9, we know that the distance between the reachable sets of $T_{1}$ and $T_{2}$ (i.e. the reachability metric) is bounded by $d_{\mathcal{B}}\left(T_{1}, T_{2}\right)$ and hence by 1 . It is easy to compute the reachable set of $T_{2}$ which is equal to $(-1,5]$. Then, from Theorem 9, we obtain that $\operatorname{Reach}\left(T_{1}\right) \subseteq[-2,6]$. The systematic computation of such approximations for nondeterministic linear systems using robust Lyapunov techniques is the focus of current research for linear [29], nonlinear [30] and hybrid systems [31].

\section{B. Deterministic Continuous Systems}

The second example we consider consists in the approximation of a high dimensional deterministic linear system of the form

$$
\left\{\begin{array}{l}
\dot{x}_{1}(t)=A_{1} x_{1}(t), x_{1}(t) \in \mathbb{R}^{100}, x_{1}(0) \in I_{1} \\
\pi_{1}(t)=C_{1} x_{1}(t), \pi_{1}(t) \in \mathbb{R}^{2}
\end{array}\right.
$$

where $I_{1}$ is a bounded polytope of $\mathbb{R}^{100}$. The unstable subspace of the system is of dimension 6. The dynamics on the 94 dimensional stable subspace was chosen at random. We want to verify that the system is safe, that is if the intersection of its reachable set with an unsafe set $\Pi_{U}$, shown in Fig. 1, is empty. We approximated this system with two different deterministic linear systems of smaller dimension.

The first approximation we considered is six dimensional and consists of simply projecting the original system on its unstable subspace. Similar to the previous example, we computed a (quadratic) bisimulation function between the two systems by solving a Lyapunov equation (see [29] for more details). Then, an upper bound of the bisimulation metric between the two systems was computed by solving the game given by Theorem 27. The second approximation is a ten dimensional approximation consisting of the projection of the original system on the subspace spanned by the eigenvectors associated to the eigenvalues with the largest real part.

Fig. 1 shows reachable sets of the hundred dimensional system, its six dimensional approximation, and its ten dimensional approximation and the associated approximation errors. We can see that the six dimensional approximation does not allow us to conclude that the system is safe, even though the original system is actually safe. However, by adding slightly more modeling detail, the ten dimensional approximation allows to conclude that the original system is safe.

The reachable sets were computed using the very recent zonotope techniques [25]. The system (Pentium 3, $700 \mathrm{MHz}$, Scilab) needed 51 seconds to compute the reachable set of the hundred dimensional system. It needed less than 1 second to process the six dimensional approximation, including the computation of the reachable set, the computation of a bisimulation function and the computation of an upper bound of the bisimulation metric. It needed about 4 seconds to process the same tasks for the ten dimensional approximation. This is strong evidence, that approximate bisimulations allow to significantly reduce the computation time of the verification process. In [29]-[31], we propose systematic methods for the computation of simulation and bisimulation functions for linear, nonlinear and hybrid systems, that can be used for reducing the complexity of most safety verification approaches for continuous and hybrid systems. More examples of application of our framework can be found in these papers.

The example also illustrates the important point that robustness simplifies verification. Indeed, if the distance between the reachable set of the original system and the set of unsafe states would have been larger then the approximation of the original system by its unstable subsystem might have been sufficient to check the safety. Generally, the more robustly safe a system is, the larger the distance from the unsafe set, resulting in larger model compression and easier safety verification.

\section{CONCLUSION}

In this paper, we have developed a framework of system approximation for metric transition systems by developing a hierarchy of metrics for reachable set inclusion, language inclusion and simulation and bisimulation relations. Our framework is compositional and captures the established exact relationships for discrete systems, and enables approximate relationships for deterministic and nondeterministic continuous systems. The exact computation of the metrics, which requires the branching distance and solving a static game, can be relaxed in a Lyapunov-like manner using simulation and bisimulations functions.

Future research includes developing algorithmic methods for computing such functions for linear, nonlinear, and hybrid systems. Even though we considered synchronous composition 
in this paper, more general composition operators will also be considered. Finally, for sophisticated verification properties expressible in temporal logics, an exciting direction emerges in understanding the relationship between approximation metrics and more robust semantics of spatial and temporal logics [35].

\section{APPENDIX}

\section{Set Valued Continuity}

Following [33], the set valued map $\mathrm{Post}^{\sigma}$ is called:

- upper semicontinuous at $q \in Q$ if for any neighborhood $V$ of $\operatorname{Post}^{\sigma}(q)$,

$\exists \eta>0$ such that $\forall q^{\prime} \in Q, d_{Q}\left(q, q^{\prime}\right) \leq \eta \Longrightarrow \operatorname{Post}^{\sigma}\left(q^{\prime}\right) \subset V$;

- lower semicontinuous at $q \in \operatorname{Supp}\left(\operatorname{Post}^{\sigma}\right)$ if for any $q^{\prime} \in \operatorname{Post}^{\sigma}(q)$ and for any sequence of elements $q_{n} \in$ $\operatorname{Supp}\left(\operatorname{Post}^{\sigma}\right)$ converging to $q$, there exists a sequence of elements $q_{n}^{\prime} \in \operatorname{Post}^{\sigma}\left(q_{n}\right)$ converging to $q^{\prime}$;

- continuous at $q \in \operatorname{Supp}\left(\mathrm{Post}^{\sigma}\right)$ if it is both upper semicontinuous and lower semicontinuous at $q$. If $\mathrm{Post}^{\sigma}$ is continuous at all $q \in \operatorname{Supp}\left(\mathrm{Post}^{\sigma}\right)$ then we say that it is continuous.

\section{Metrics, Hausdorff Distance}

Definition 22 (Metric): A metric on a set $E$ is a positive function $d: E \times E \rightarrow \mathbb{R} \cup\{+\infty\}$, such that the three following properties hold:

1) for all $e_{1} \in E, e_{2} \in E, e_{3} \in E, d\left(e_{1}, e_{3}\right) \leq d\left(e_{1}, e_{2}\right)+$ $d\left(e_{2}, e_{3}\right)$

2) for all $e_{1} \in E, e_{2} \in E, d\left(e_{1}, e_{2}\right)=0 \Longleftrightarrow e_{1}=e_{2}$;

3) for all $e_{1} \in E, e_{2} \in E, d\left(e_{1}, e_{2}\right)=d\left(e_{2}, e_{1}\right)$.

We say that $(E, d)$ is a metric space. If the second property is replaced by $e_{1}=e_{2} \Longrightarrow d\left(e_{1}, e_{2}\right)=0$ then $d$ is called a pseudo-metric. If the third property is dropped, then $d$ is called a directed metric.

A metric on a set $E$ induces a natural metric on the set of subsets of $E$ known as the Hausdorff distance (see, e.g., [36]).

Definition 23 (Hausdorff Distance): Let $E_{1}$ and $E_{2}$ be two subsets of $E$. The directed Hausdorff distance associated to the metric $d$ is defined by

$$
h \rightarrow\left(E_{1}, E_{2}\right)=\sup _{e_{1} \in E_{1}} \inf _{e_{2} \in E_{2}} d\left(e_{1}, e_{2}\right) .
$$

The Hausdorff distance associated to the metric $d$ is then

$$
h\left(E_{1}, E_{2}\right)=\max \left(h \rightarrow\left(E_{1}, E_{2}\right), h \rightarrow\left(E_{2}, E_{1}\right)\right) .
$$

We have the following classical theorem.

Theorem 29: The (directed) Hausdorff distance is a (directed) pseudo-metric on the set of subsets of $E$ and

$$
\begin{aligned}
& h^{\rightarrow}\left(E_{1}, E_{2}\right)=0 \quad \text { if and only if } \quad \operatorname{cl}\left(E_{1}\right) \subseteq \operatorname{cl}\left(E_{2}\right) \\
& h\left(E_{1}, E_{2}\right)=0 \text { if and only if } \operatorname{cl}\left(E_{1}\right)=\operatorname{cl}\left(E_{2}\right)
\end{aligned}
$$

where $\operatorname{cl}\left(E_{i}\right)$ denotes the closure of the set $E_{i}$.

\section{REFERENCES}

[1] R. Milner, Communication and Concurrency. Upper Saddle River, NJ: Prentice-Hall, 1989.

[2] E. M. Clarke, O. Grumberg, and D. A. Peled, Model Checking. Cambridge, MA: MIT Press, 2000.
[3] J. R. Burch, E. M. Clarke, K. L. McMillan, D. L. Dill, and L. J. Hwang, "Symbolic model checking: $10^{20}$ states and beyond," in Proc. IEEE Symp. Logic Comp. Sci., 1990, pp. 1-33.

[4] R. Alur, T. A. Henzinger, G. Lafferriere, and G. J. Pappas, "Discrete abstractions of hybrid systems," Proc. IEEE, vol. 88, no. 7, pp. 971-984, Jul. 2000.

[5] G. J. Pappas, "Bisimilar linear systems," Automatica, vol. 39, no. 12, pp. 2035-2047, Dec. 2003.

[6] P. Tabuada and G. J. Pappas, "Bisimilar control affine systems," Syst. Control Lett., vol. 52, pp. 49-58, 2004.

[7] A. van der Schaft, "Equivalence of dynamical systems by bisimulation," IEEE Trans. Autom. Control, vol. 49, no. 12, pp. 2160-2172, Dec. 2004.

[8] E. Haghverdi, P. Tabuada, and G. J. Pappas, "Bisimulation relations for dynamical, control, and hybrid systems," Theor. Comp. Sc., vol. 342, no. 2-3, pp. 229-261, September 2005.

[9] A. A. Julius and A. J. van der Schaft, "A behavioral framework for compositionality: Linear systems, discrete event systems and hybrid systems," in Proc. MTNS, 2004.

[10] G. Pola, A. J. van der Schaft, and M. D. Di Benedetto, "Bisimulation theory for switching linear systems," in Proc. IEEE Conf. Decision Control, 2004, pp. 555-569.

[11] A. C. Antoulas, D. C. Sorensen, and S. Gugercin, "A survey of model reduction methods for large-scale systems," Contemp. Math., vol. 280, pp. 193-219, 2000.

[12] P. Caspi and A. Benveniste, "Toward an approximation theory for computerized control," in Embedded Software. New York: Springer-Verlag, 2002, vol. 2491, LNCS, pp. 294-304.

[13] A. Di Pierro, C. Hankin, and H. Wiklicky, "Quantitative relations and approximate process equivalences," in Proc. CONCUR 2003, 2003, vol. 2761, LNCS, pp. 508-522.

[14] F. van Breugel, M. Mislove, J. Ouaknine, and J. Worrell, "An intrinsic characterization of approximate probabilistic bisimilarity," in Foundations of Software Science and Computation Structures. New York: Springer-Verlag, 2003, vol. 2620, LNCS, pp. 200-215.

[15] J. Desharnais, V. Gupta, R. Jagadeesan, and P. Panangaden, "Metrics for labelled markov processes," Theor. Comp. Sci., vol. 318, no. 3, pp. 323-354, June 2004.

[16] L. de Alfaro, M. Faella, and M. Stoelinga, "Linear and branching metrics for quantitative transition systems," in Proc. ICALP'04, 2004, vol. 3142, LNCS, pp. 1150-1162.

[17] P. C. Kanellakis and S. A. Smolka, "CCS expressions, finite-state processes, and three problems of equivalence," Inform. Comput., vol. 86, pp. 43-68, 1990

[18] R. Alur, T. Dang, and F. Ivancic, "Reachability analysis of hybrid systems via predicate abstraction," in Proc. HSCC, 2002, vol. 2289, LNCS, pp. 35-48.

[19] A. Tiwari and G. Khanna, "Series of abstractions for hybrid automata," in Proc. HSCC, 2002, vol. 2289, LNCS, pp. 465-478.

[20] E. Asarin, O. Bournez, T. Dang, and O. Maler, "Approximate reachability analysis of piecewise linear dynamical systems," in Proc. HSCC, 2000, vol. 1790, LNCS, pp. 21-31.

[21] E. Asarin, T. Dang, and A. Girard, "Reachability of non-linear systems using conservative approximations," in Proc. HSCC, 2003, vol. 2623, LNCS, pp. 22-35.

[22] A. Chutinan and B. H. Krogh, "Verification of polyhedral invariant hybrid automata using polygonal flow pipe approximations," in Proc. HSCC, 1999, vol. 1569, LNCS, pp. 76-90.

[23] A. Kurzhanski and P. Varaiya, "Ellipsoidal tehcniques for reachability analysis," in Proc. HSCC, 2000, vol. 1790, LNCS.

[24] I. Mitchell and C. Tomlin, "Level set methods for computation in hybrid systems," in proc. HSCC, 2000, vol. 1790, LNCS.

[25] A. Girard, "Reachability of uncertain linear systems using zonotopes," in Proc. HSCC, 2005, vol. 3414, LNCS, pp. 291-305.

[26] S. Prajna and A. Jadbabaie, "Safety verification of hybrid systems using barrier certificates," in Proc. HSCC, 2004, vol. 2993, LNCS, pp. 477-492.

[27] Z. Han and B. H. Krogh, "Reachability of hybrid control systems using reduced-order models," in Proc. Amer. Control Conf., 2004, pp. 1183-1189.

[28] E. Asarin and T. Dang, "Abstraction by projection and application to multi-affine systems," in Proc. HSCC, 2004, vol. 2993, LNCS, pp. $32-47$.

[29] A. Girard and G. J. Pappas, "Approximate bisimulations for constrained linear systems," in Proc. Conf. Decision Control Euro. Control Conf., 2005, pp. 4700-4705. 
[30] A. Girard and G. J. Pappas, "Approximate bisimulations for nonlinear dynamical systems," in Proc. Conf. Decision Control Euro. Control Conf., 2005, pp. 684-689.

[31] A. Girard, A. A. Julius, and G. J. Pappas, "Approximate simulation relations for hybrid systems," in Proc. ADHS, 2006, pp. 106-111.

[32] E. W. Weisstein et al., "Locally compact," From MathWorld-A Wolfram Web Resource 2005.

[33] J. P. Aubin, Viability Theory. Boston, MA: Birkhäuser, 1991.

[34] A. Girard and G. J. Pappas, Approximation metrics for discrete and continuous systems Dept. CIS, Univ. Pennsylvania, Philadelphia, PA, Tech. Rep. MS-CIS-05-10, May 2005.

[35] J. M. Davoren, V. Coulthard, N. Markey, and T. Moor, "Non-deterministic temporal logics for general flow systems," in Proc. HSCC, 2004, vol. 2993, LNCS, pp. 280-295.

[36] G. Ewald, Combinatorial Convexity and Algebraic Geometry. New York: Springer-Verlag, 1996.

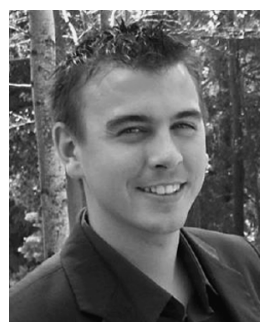

Antoine Girard received the Dip.Ing. from the Ecole Nationale Supérieure d'Informatique et de Mathématiques Appliquées de Grenoble, Grenoble, France, the M.S. degree in applied mathematics from the Université Joseph Fourier, Grenoble, France, and the Ph.D. degree in applied mathematics from the Institut National Polytechnique de Grenoble, France, in 2001 and 2004, respectively.

From October 2004 to December 2005, he was a Postdoctoral Researcher with the Department of Electrical and Systems Engineering of the University of Pennsylvania, Philadelphia, and from January to August 2006, he was a Postdoctoral Researcher at the Verimag Laboratory, Grenoble, France. Since September 2006, he has been an Assistant Professor at the Université Joseph
Fourier, Grenoble, France. His research interests include algorithmic analysis and approximation theory of discrete, continuous, and hybrid systems, as well as hierarchical and multiscale approaches to control.

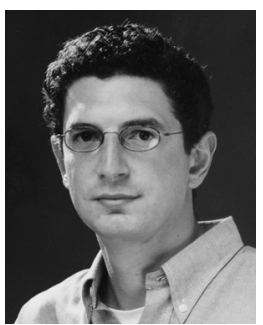

George J. Pappas (S'91-M'98-SM'04) received the $\mathrm{Ph} . \mathrm{D}$. degree in electrical engineering and computer sciences from the University of California, Berkeley, in 1998.

He is currently an Associate Professor in the Department of Electrical and Systems Engineering, and the Director of the GRASP Laboratory. He also holds secondary appointments in the Departments of Computer and Information Sciences, and Mechanical Engineering and Applied Mechanics. He has published over one hundred articles in the areas of hybrid systems, hierarchical control systems, distributed control systems, nonlinear control systems, and geometric control theory, with applications to robotics, unmanned aerial vehicles, and biomolecular networks. He coedited Hybrid Systems: Computation and Control (New York: Springer-Verlag, 2004, ser. Lecture Notes in Computer Science).

Dr. Pappas was the recipient of a National Science Foundation (NSF) Career Award in 2002, as well as the 2002 NSF Presidential Early Career Award for Scientists and Engineers (PECASE). He received the 1999 Eliahu Jury Award for Excellence in Systems Research from the Department of Electrical Engineering and Computer Sciences, University of California at Berkeley. His and his students' papers were finalists for the Best Student Paper Award at the IEEE Conference on Decision and Control (1998, 2001, and 2004), and the American Control Conference (2001 and 2004). He is currently serving as an Associate Editor for the IEEE TRANSACTIONS ON AUTOMATIC CONTROL. 